Research Article

Special Issue in memory of Abe Sklar

Open Access

Thimo M. Kasper, Sebastian Fuchs, and Wolfgang Trutschnig*

\title{
On convergence of associative copulas and related results
}

https://doi.org/10.1515/demo-2021-0114

Received May 27, 2021; accepted July 22, 2021

\begin{abstract}
Triggered by a recent article establishing the surprising result that within the class of bivariate Archimedean copulas $\mathcal{C}_{\text {ar }}$ different notions of convergence - standard uniform convergence, convergence with respect to the metric $D_{1}$, and so-called weak conditional convergence - coincide, in the current contribution we tackle the natural question, whether the obtained equivalence also holds in the larger class of associative copulas $\mathcal{C}_{\mathrm{a}}$. Building upon the fact that each associative copula can be expressed as (finite or countably infinite) ordinal sum of Archimedean copulas and the minimum copula $M$ we show that standard uniform convergence and convergence with respect to $D_{1}$ are indeed equivalent in $\mathcal{C}_{\mathrm{a}}$. It remains an open question whether the equivalence also extends to weak conditional convergence. As by-products of some preliminary steps needed for the proof of the main result we answer two conjectures going back to Durante et al. and show that, in the language of Baire categories, when working with $D_{1}$ a typical associative copula is Archimedean and a typical Archimedean copula is strict.
\end{abstract}

Keywords: associative copulas, Archimedean copulas, weak convergence, Baire category

MSC: 62H05, 60E05, 54E52

\section{Introduction}

Various different notions of convergence in the family of bivariate copulas $\mathcal{C}$ have been considered in the literature: The standard uniform metric $d_{\infty}$ is probably the most common choice; since, however, $d_{\infty}$ is not capable of distinguishing independence and complete dependence (or, in the words of [24], $d_{\infty}$ does not 'distinguish between different types of statistical dependence') the stronger metric $D_{1}$ was introduced in [36]. Letting $K_{A}(\cdot, \cdot), K_{B}(\cdot, \cdot)$ denote Markov kernels (regular conditional distributions) of the copulas $A$ and $B$, respectively, the metric $D_{1}$ is defined by

$$
D_{1}(A, B)=\int_{[0,1]^{2}}\left|K_{A}(x,[0, y])-K_{B}(x,[0, y])\right| \mathrm{d} \lambda_{2}(x, y) .
$$

According to [36], $D_{1}$ is a metrization of the strong operator topology (of the Markov operators induced by the copulas, see [24, 29] for more information on the strong operator topology and the one-to-one correspondence between bivariate copulas and Markov operators), convergence with respect to $D_{1}$ implies convergence with respect to $d_{\infty}$ (but not vice versa) and, setting $\zeta_{1}(A):=3 D_{1}(A, \Pi)$ yields a dependence measure $\zeta_{1}$ fulfilling that $\zeta_{1}(A)$ is maximal if and only if $A$ is completely dependent.

Thimo M. Kasper: Department for Mathematics, University of Salzburg, E-mail: thimo.kasper@sbg.ac.at Sebastian Fuchs: Department for Mathematics, University of Salzburg, E-mail: sebastian.fuchs@sbg.ac.at ^Corresponding Author: Wolfgang Trutschnig: Department for Mathematics, University of Salzburg, E-mail: wolfgang.trutschnig@sbg.ac.at 
Sticking to Markov kernels/conditional distributions and considering weak convergence of almost all conditional distributions of copulas results in the notion of weak conditional convergence which was introduced recently in [17]. Weak conditional convergence implies convergence with respect to $D_{1}$, and it is straightforward to construct examples illustrating that the reverse implication is wrong (again see [17]). When working within the subclass of absolutely continuous copulas other natural notions of convergence are the total variation distance TV (which, in the absolutely continuous setting coincides with the $L_{1}$-distance of the densities) and the Kullback-Leibler divergence KL (see, e.g., [35] and the references therein). In case the copula densities are greater than 0 almost everywhere then according to [26] we have the following interrelation, where $a \Rightarrow b$ indicates the convergence with respect to $a$ implies convergence with respect to $b$ :

$$
K L \Rightarrow T V \Rightarrow D_{1} \Rightarrow d_{\infty}
$$

As mentioned above, the topologies induced by $d_{\infty}, D_{1}$ and weak conditional convergence on $\mathcal{C}$ do not coincide: the topology induced by weak conditional convergence is strictly finer than the one induced by $D_{1}$, which, in turn is strictly finer than the one induced by $d_{\infty}$. According to [17], however, on large classes like the family of Archimedean copulas as well as on the family of Extreme Value copulas, all three topologies do coincide. Considering that the family of Archimedean copulas is dense in the family of associative copulas with respect to $d_{\infty}$ (see $\left.[18,19]\right)$ and that every associative copula admits a so-called ordinal sum representation (see [23]) in terms of finitely or countably infinitely many Archimedean copulas and $M$ it is natural to ask, whether the three notions of convergence are also equivalent on the family $\mathcal{C}_{a}$ of all associative copulas. In the current contribution we provide a partial affirmative answer and show that on $\mathcal{C}_{\mathrm{a}}$ convergence with respect to $d_{\infty}$ and $D_{1}$ are indeed equivalent. The question, whether weak conditional convergence is equivalent too, remains open - we have neither been able to prove equivalence nor to construct a counterexample.

Although the main result of the paper is the equivalence of $d_{\infty}$ and $D_{1}$ on $\mathcal{C}_{\mathrm{a}}$ several auxiliary results on denseness of the class of strict and the class of non-strict Archimedean copulas in $\left(\mathcal{C}_{\mathrm{a}}, D_{1}\right)$ produce nice by-products in so far as we are able to answer two conjectures going back to Durante et al. in [6] and show that, in the language of Baire categories, when working with $D_{1}$ a 'typical' associative copula is Archimedean and a 'typical' Archimedean copula is strict.

The remainder of this paper is organized as follows: Section 2 contains notation and preliminaries which will be used in the sequel. In Section 3 we first prove the fact that the family of all strict and the family of all non-strict Archimedean copulas are dense in $\left(\mathcal{C}_{a}, D_{1}\right)$ by following the procedure studied in [19] for $d_{\infty}$, and then show the afore-mentioned Baire category results. Finally, Section 4 focuses on convergence of associative copulas and establishes the main result in several steps. Some examples and graphics illustrate the chosen approach.

\section{Notation and preliminaries}

In the sequel we will let $\mathcal{C}$ denote the family of all bivariate copulas. For each copula $C$ the corresponding doubly stochastic measure will be denoted by $\mu_{C}$, i.e. $\mu_{C}([0, x] \times[0, y])=C(x, y)$ for all $x, y \in[0,1]$. For more background on copulas and doubly stochastic measures we refer to $[8,27]$.

As copulas can be seen as binary operations on $[0,1]^{2}$ associativity is of particular interest, that is, $C \in \mathcal{C}$ is called associative if for all $x, y, z \in[0,1]$ we have

$$
C(C(x, y), z)=C(x, C(y, z)) .
$$

$\mathcal{C}_{\mathrm{a}}$ denotes the class of all associative copulas. Associative copulas are closely related to triangular norms. In fact, according to [25] a copula $C$ is a triangular norm if and only if $C \in \mathcal{C}_{\mathrm{a}}$ whereas a triangular norm $T$ is a copula if and only if it is Lipschitz continuous with Lipschitz constant 1 (see, for example, $[1,18]$ ).

The standard uniform metric $d_{\infty}$ on $\mathcal{C}$ is defined by

$$
d_{\infty}\left(C_{1}, C_{2}\right):=\max _{(x, y) \in[0,1]^{2}}\left|C_{1}(x, y)-C_{2}(x, y)\right| .
$$


It is well known that the metric space ( $\left.\mathcal{C}, d_{\infty}\right)$ is compact and that pointwise and uniform convergence of a sequence of copulas $\left(C_{n}\right)_{n \in \mathbb{N}}$ are equivalent (see $\left.[8,37]\right)$.

For every metric space $(S, d)$ the Borel $\sigma$-field on $S$ will be denoted by $\mathcal{B}(S)$. In what follows Markov kernels will play a prominent role. A Markov kernel from $\mathbb{R}$ to $\mathbb{R}$ is a mapping $K: \mathbb{R} \times \mathcal{B}(\mathbb{R}) \rightarrow[0,1]$ such that for every fixed $E \in \mathcal{B}(\mathbb{R})$ the mapping $x \mapsto K(x, E)$ is (Borel-)measurable and for every fixed $x \in \mathbb{R}$ the mapping $E \mapsto K(x, E)$ is a probability measure. Given two real-valued random variables $X, Y$ on a probability space $(\Omega, \mathcal{A}, \mathbb{P})$ and letting $\mathbb{1}_{E}$ denote the characteristic function of a set $E \subseteq \mathbb{R}$ we say that a Markov kernel $K$ is a regular conditional distribution of $Y$ given $X$ if $K(X(\omega), E)=\mathbb{E}\left(\mathbb{1}_{E} \circ Y \mid X\right)(\omega)$ holds $\mathbb{P}$-almost surely for every $E \in \mathcal{B}(\mathbb{R})$. It is well-known that for $X, Y$ as above, a regular conditional distribution of $Y$ given $X$ always exists and is unique for $\mathbb{P}^{X}$-a.e. $x \in \mathbb{R}$ whereby $\mathbb{P}^{X}$ denotes the push-forward of $\mathbb{P}$ via $X$, i.e., $\mathbb{P}^{X}(E)=$ $\mathbb{P}\left(X^{-1}(E)\right.$ ) for every $E \in \mathcal{B}(\mathbb{R})$ (see, e.g., [15, 20]). In case $(X, Y)$ has distribution function $C \in \mathcal{C}$ we will let $K_{C}:[0,1] \times \mathcal{B}([0,1]) \rightarrow[0,1]$ denote (a version of) the regular conditional distribution of $Y$ given $X$ and refer to it as Markov kernel of $C$. Defining the $x$-section of a set $G \in \mathcal{B}\left([0,1]^{2}\right)$ by $G_{x}:=\{y \in[0,1]:(x, y) \in G\}$ we have the following disintegration formula (see $[15,20]$ )

$$
\int_{[0,1]} K_{C}\left(x, G_{x}\right) d \mathbb{P}^{X}(x)=\mu_{C}(G)
$$

hence, in particular,

$$
\int_{[0,1]} K_{C}(x, E) \mathrm{d} \mathbb{P}^{X}(x)=\int_{[0,1]} K_{C}(x, E) \mathrm{d} \lambda(x)=\lambda(E)
$$

for every $E \in \mathcal{B}([0,1])$, whereby $\lambda$ denotes the Lebesgue measure on $\mathbb{R}$. For more information on conditional expectation and general disintegration we refer to $[15,20]$.

Following [8] the rightside upper Dini derivative $\bar{\partial}_{1}^{+} C:[0,1) \times[0,1] \rightarrow[0,1]$ of a copula $C$ with respect to the first coordinate is defined by

$$
\bar{\partial}_{1}^{+} C(x, y):=\limsup _{h \rightarrow 0+} \frac{C(x+h, y)-C(x, y)}{h} \in[0,1] .
$$

As shown in [7], the map $y \mapsto \bar{\partial}_{1}^{+} C(x, y)$ is non-decreasing which we will use subsequently to clarify the relation between Markov kernel and rightside upper Dini derivative:

Lemma 2.1. Let $C$ be an arbitrary copula. Then

$$
\bar{\partial}_{1}^{+} C(x, y)=K_{C}(x,[0, y])
$$

for $\lambda_{2}$-a.e. $(x, y) \in(0,1)^{2}$.

Proof. Letting $\partial_{i}$ denote the partial derivative with respect to the $i$-th coordinate it is well-known (see, e.g., $[8,33])$ that for every $y \in[0,1]$ there exists a set $\Lambda_{y} \in \mathcal{B}([0,1])$ of full measure such that for all $x \in \Lambda_{y}$ we have that $\partial_{1} C(x, y)$ exists and

$$
\bar{\partial}_{1}^{+} C(x, y)=\partial_{1} C(x, y)=K_{C}(x,[0, y])
$$

holds. Then $\Lambda:=\bigcap_{y \in(0,1) \cap \mathbb{Q}} \Lambda_{y} \in \mathcal{B}([0,1])$ is a set of full measure and for all $x \in \Lambda, y \in(0,1) \cap \mathbb{Q}$ we have

$$
\bar{\partial}_{1}^{+} C(x, y)=K_{C}(x,[0, y]) .
$$

As for $x \in \Lambda, y \in(0,1)$ we have $K_{C}(x,[0, y]) \geq \bar{\partial}_{1}^{+} C(x, y)$ it suffices to show that

$$
\Gamma:=\left\{(x, y) \in[0,1]^{2}: x \in \Lambda, K_{C}(x,[0, y])>\bar{\partial}_{1}^{+} C(x, y)\right\}
$$

is a $\lambda_{2}$-null set which can be done as follows: First, note that $(x, y) \mapsto K_{C}(x,[0, y])$ as well as $(x, y) \mapsto$ $K_{C}(x,[0, y))$ are measurable functions. Second, for $x \in \Lambda, y \in[0,1]$ we have $K_{C}(x,[0, y])>\bar{\partial}_{1}^{+} C(x, y) \Leftrightarrow$ 
$K_{C}(x,[0, y])>K_{C}(x,[0, y))$ whence

$$
\begin{aligned}
\Gamma & =\left\{(x, y) \in \Lambda \times[0,1]: K_{C}(x,[0, y))<K_{C}(x,[0, y])\right\} \\
& =\left\{(x, y) \in[0,1]^{2}: K_{C}(x,[0, y))<K_{C}(x,[0, y])\right\} \cap(\Lambda \times[0,1])
\end{aligned}
$$

which is measurable. As for $x \in \Lambda$ we have $\Gamma_{x}$ is at most countably infinite, applying disintegration yields $\lambda_{2}(\Gamma)=0$.

The following consequence of Lemma 2.1 is immediate:

Corollary 2.2. Let $C_{1}, C_{2} \in \mathcal{C}$ and $U, V \subseteq[0,1]$ with $U$ open. If $C_{1}$ is identical to $C_{2}$ on $U \times V \subseteq[0,1]^{2}$ then

$$
K_{C_{1}}(x,[0, y])=K_{C_{2}}(x,[0, y])
$$

for $\lambda_{2}$-a.e. $(x, y) \in U \times V$.

A copula $C$ is called completely dependent if there exists a $\lambda$-preserving transformation $h:[0,1] \rightarrow[0,1]($ i.e., a transformation fulfilling $\lambda\left(h^{-1}(E)\right)=\lambda(E)$ for every $\left.E \in \mathcal{B}([0,1])\right)$ such that $K(x, E):=\mathbb{1}_{E}(h(x))$ is a Markov kernel of $C$. The class of all completely dependent copulas will be denoted by $\mathcal{C}_{\text {cd }}$.

For two copulas $C_{1}, C_{2}$ the so-called Markov product or star product $C_{1} * C_{2}$ is defined by

$$
\left(C_{1} * C_{2}\right)(x, y):=\int_{[0,1]} \partial_{2} C_{1}(x, t) \cdot \partial_{1} C_{2}(t, y) \mathrm{d} \lambda(t) .
$$

The star-product is always a copula (see [5]) and according to results of [38] a Markov kernel of $C_{1} * C_{2}$ is given by

$$
\left(K_{C_{1}} \circ K_{C_{2}}\right)(x, E):=\int_{[0,1]} K_{C_{2}}(y, E) K_{C_{1}}(x, \mathrm{~d} y) .
$$

Subsequently we will make use of the following properties of the star product: First, $C \in \mathcal{C}$ is completely dependent if and only if there exists a copula $B$ such that the identity $B * C=M$ holds, and second, if $B \in \mathcal{C}$ and if the sequence of copulas $\left(C_{n}\right)_{n \in \mathbb{N}}$ converges uniformly to another copula $C$ then $\left(B * C_{n}\right)_{n \in \mathbb{N}}$ converges uniformly to $B * C$. For more properties of the class $\mathcal{C}_{\text {cd }}$ and the star product we refer to $[22,36]$ and $[5,14]$, respectively.

Considering Markov kernels allows to define stronger metrics than the standard uniform one:

$$
\begin{aligned}
D_{1}\left(C_{1}, C_{2}\right) & :=\int_{[0,1]^{2}}\left|K_{C_{1}}(x,[0, y])-K_{C_{2}}(x,[0, y])\right| \mathrm{d} \lambda_{2}(x, y), \\
D_{2}^{2}\left(C_{1}, C_{2}\right) & :=\int_{[0,1]^{2}}\left(K_{C_{1}}(x,[0, y])-K_{C_{2}}(x,[0, y])\right)^{2} \mathrm{~d} \lambda_{2}(x, y), \\
D_{\infty}\left(C_{1}, C_{2}\right) & :=\sup _{y \in[0,1]} \int_{[0,1]}\left|K_{C_{1}}(x,[0, y])-K_{C_{2}}(x,[0, y])\right| \mathrm{d} \lambda(x) .
\end{aligned}
$$

In [36] it was shown that $D_{1}, D_{2}, D_{\infty}$ are three metrics generating the same topology on $\mathcal{C}$. In what follows we will primarily work with $D_{1}$ and refer to $[9,36]$ for more information on $D_{2}$ and $D_{\infty}$. The metric space $\left(\mathcal{C}, D_{1}\right)$ is complete and separable but not compact (again see [36]).

An even stronger notion of convergence involving Markov kernels is introduced and studied in [17]: Let $C, C_{1}, C_{2}, \ldots$ be copulas with corresponding Markov kernels $K_{C}, K_{C_{1}}, K_{C_{2}}, \ldots$ Then $\left(C_{n}\right)_{n \in \mathbb{N}}$ is said to converge weakly conditional to $C$ if and only if for $\lambda$-almost every $x \in[0,1]$ the sequence $\left(K_{C_{n}}(x, \cdot)\right)_{n \in \mathbb{N}}$ of probability measures on $\mathcal{B}([0,1])$ converges weakly to the probability measure $K_{C}(x, \cdot)$ (in short, $C_{n} \stackrel{w c c}{\longrightarrow} C$, where 'wcc' stands for 'weak conditional convergence'). The following interrelation of the afore-mentioned modes of convergence holds:

$$
\text { weak conditional convergence } \Rightarrow \text { convergence w.r.t. } D_{1} \Rightarrow \text { convergence w.r.t. } d_{\infty} \text {. }
$$

For counterexamples for the reverse implications again see [17] and [36]. 


\section{The interrelation of Archimedean and associative copulas with respect to $D_{1}$}

Triggered by [19], where the authors prove that the class of Archimedean copulas is dense in the class of associative copulas with respect to the uniform metric $d_{\infty}$, in what follows we show the result w.r.t. $D_{1}$ and, as a by-product, answer two open questions posed in [6] regarding Baire category results for Archimedean copulas. In Section 4 we then exploit the proven denseness to derive surprising convergence results of associative copulas with respect to $D_{1}$.

An Archimedean copula $A=A_{\varphi}$ is a copula induced by a convex and strictly decreasing function $\varphi$ : $[0,1] \rightarrow[0, \infty]$ with $\varphi(1)=0$, called generator, via

$$
A(x, y)=\varphi^{-}(\varphi(x)+\varphi(y)), \quad x, y \in[0,1] .
$$

Thereby $\varphi^{-}:[0, \infty] \rightarrow[0,1]$ denotes the pseudoinverse of $\varphi$, defined by

$$
\varphi^{-}(x):=\left\{\begin{array}{ll}
\varphi^{-1}(x) & \text { if } x \in[0, \varphi(0+)) \\
0 & \text { if } x \geq \varphi(0+)
\end{array},\right.
$$

where $\varphi\left(x_{ \pm}\right):=\lim _{t \rightarrow x^{ \pm}} \varphi(t)$ is the respective one-sided limit. We refer to $A$ as the Archimedean copula induced by $\varphi$; in case of $\varphi(0+)=\infty, A$ is called strict and non-strict otherwise. In the sequel $\mathcal{C}_{\text {ar }}$ denotes the class of all Archimedean copulas, $\mathcal{C}_{\mathrm{ar}}^{\mathrm{s}}$ the subclass of strict Archimedean copulas and $\mathcal{C}_{\mathrm{ar}}^{\mathrm{s}}$ the subclass of nonstrict Archimedean copulas. Since generators are only unique up to a multiplicative constant we will from now on also assume (without explicit reference) that the generator is normalized in the sense that $\varphi\left(\frac{1}{2}\right)=1$ holds. With this convention there is a one-to-one correspondence between generators and induced copulas.

In what follows we let $D^{+} \varphi(x)\left(D^{-} \varphi(x)\right)$ denote the right-hand (left-hand) derivative of $\varphi$ at $x \in(0,1)$. By convexity, $\varphi$ is differentiable outside a countable subset of $(0,1)$, i.e. $D^{+} \varphi(x)=D^{-} \varphi(x)$ holds for all but at most countably many $x \in(0,1)$ and $D^{+} \varphi$ is non-decreasing and right-continuous. According to [16, 31] we additionally have $D^{-} \varphi(x)=D^{+} \varphi(x-)$ for every $x \in(0,1)$. Letting $\operatorname{Cont}\left(D^{+} \varphi\right) \subseteq(0,1)$ denote the set of all continuity points of $D^{+} \varphi$ in $(0,1)$ it follows that $[0,1] \backslash \operatorname{Cont}\left(D^{+} \varphi\right)$ is at most countably infinite and thus has Lebesgue measure 0 . Setting $D^{+} \varphi(0)=-\infty$ in case of strict $\varphi$ as well as $D^{+} \varphi(1)=0$ (for strict and non-strict $\varphi$ ) allows to view $D^{+} \varphi$ as non-decreasing and right-continuous function on the full unit interval.

The Kendall distribution function of an Archimedean copula $A$ with generator $\varphi$ is given by (see, e.g., [13])

$$
F_{A}^{K}(x)=x-\frac{\varphi(x)}{D^{+} \varphi(x)} .
$$

Following $[10,27]$ for every $t \in[0,1]$ we define the $t$-level function $f^{t}:[t, 1] \rightarrow[0,1]$ by $f^{t}(x):=\varphi^{-1}(\varphi(t)-$ $\varphi(x))$ so that for $t>0$

$$
\operatorname{graph}\left(f^{t}\right)=\left\{\left(x, f^{t}(x)\right): x \in[t, 1]\right\}=\left\{(x, y) \in[0,1]^{2}: C(x, y)=t\right\}=: L_{t}
$$

holds since $C$ is strictly increasing coordinatewise above $C^{-1}(\{0\})$. If $\varphi$ is strict then according to [10]

$$
K_{A}(x,[0, y])= \begin{cases}\frac{D^{+} \varphi(x)}{D^{+} \varphi(A(x, y))} & \text { if } x \in(0,1) \\ 1 & \text { if } x \in\{0,1\}\end{cases}
$$

is (a version of) the Markov kernel of $A$, for non-strict $\varphi$ a version is given by

$$
K_{A}(x,[0, y])= \begin{cases}0 & \text { if } x \in(0,1), y<f^{0}(x) \\ \frac{D^{+} \varphi(x)}{D^{+} \varphi(A(x, y))} & \text { if } x \in(0,1), y \geq f^{0}(x) \\ 1 & \text { if } x \in\{0,1\}\end{cases}
$$


According to [17] all three afore-mentioned modes of convergence coincide within $\mathcal{C}_{\text {ar }}$, that is, a sequence of Archimedean copulas $\left(A_{n}\right)_{n \in \mathbb{N}}$ converges uniformly to some $A \in \mathcal{C}_{\text {ar }}$ if and only if $\left(A_{n}\right)_{n \in \mathbb{N}}$ converges to $A$ with respect to $D_{1}$ if and only if $\left(A_{n}\right)_{n \in \mathbb{N}}$ converges to $A$ weakly conditional (as $\left.n \rightarrow \infty\right)$.

Archimedean copulas play a central role in our study since, together with the Fréchet-Hoeffding upper bound $M$, they form the building blocks of associative copulas: According to [23] (see also [1]) every associative copula $C$ admits an ordinal sum representation in the following sense: there exists some index set $I \subseteq \mathbb{N}$, a partition $\left\{\left[a_{i}, b_{i}\right]\right\}_{i \in I}$ of $[0,1]$ and $\left(A_{i}\right)_{i \in I}$ a sequence of copulas such that $A_{i} \in \mathcal{C}_{\mathrm{ar}} \cup\{M\}$ and

$$
C(x, y)=\left\{\begin{array}{ll}
a_{i}+\left(b_{i}-a_{i}\right) A_{i}\left(\frac{x-a_{i}}{b_{i}-a_{i}}, \frac{y-a_{i}}{b_{i}-a_{i}}\right) & \text { if }(x, y) \in\left(a_{i}, b_{i}\right)^{2} . \\
M(x, y) & \text { otherwise }
\end{array} .\right.
$$

In this case we write $C=\left(\left\langle a_{i}, b_{i}, A_{i}\right\rangle\right)_{i \in I}$. Notice that, contrary to literature, the above definition explicitly exposes (shrunk versions of) $M$ along diagonal blocks. Defining the affine transformation $T_{i}:\left[a_{i}, b_{i}\right] \rightarrow$ $[0,1], x \mapsto \frac{x-a_{i}}{b_{i}-a_{i}}$ and its inverse $T_{i}^{-1}:[0,1] \rightarrow\left[a_{i}, b_{i}\right], x \mapsto a_{i}+\left(b_{i}-a_{i}\right) x$ allows to write

$$
C(x, y)=T_{i}^{-1}\left(A_{i}\left(T_{i}(x), T_{i}(y)\right)\right)
$$

for every $(x, y) \in\left(a_{i}, b_{i}\right)^{2}$. We will also work with the pre-image of $T_{i}$ and also denote it by $T_{i}^{-1}$ since no confusion will arise.

In this paper a finite associative copula is by definition an associative copula with finite index set $I$. Moreover, a subinterval $\left(a_{i}, b_{i}\right)$ appearing in the ordinal sum representation of an associative copula $C$ will also be simply called subinterval of $C$.

We start with the following Lemma stating that any non-strict Archimedean copula can be approximated arbitrarily well by strict Archimedean copulas with respect to $D_{1}$ and vice versa:

Theorem 3.1. $\mathcal{C}_{\text {ar }}^{\mathrm{S}}$ and $\mathrm{C}_{\text {ar }}^{-\mathcal{S}}$ are dense in $\left(\mathcal{C}_{a r}, D_{1}\right)$.

Proof. We only prove denseness of strict Archimedean copulas, the non-strict case follows in a similar manner. Fix $\varepsilon>0$ and let $A_{\neg \mathrm{s}}$ have non-strict generator $\varphi_{\neg \text { s. }}$ In [19] it is shown that for every $n \in \mathbb{N}$ there exists a strict generator $\varphi_{\mathrm{s}}^{n}$ with $\varphi_{\mathrm{s}}^{n}=\varphi_{\neg \mathrm{s}}$ on $\left(\frac{1}{n}, 1\right]$ such that the corresponding strict Archimedean copula $A_{\mathrm{s}}^{n}$ fulfills $d_{\infty}\left(A_{\neg s}, A_{\mathrm{s}}^{n}\right) \leq \frac{1}{n}$. Letting $n \rightarrow \infty$ we obtain pointwise convergence of $\varphi_{\mathrm{s}}^{n} \rightarrow \varphi_{\neg \mathrm{s}}$ on $(0,1]$ and using [17, Theorem 4.2] we even have weak conditional convergence of $\left(A_{\mathrm{s}}^{n}\right)_{n \in \mathbb{N}}$ implying $D_{1}\left(A_{\mathrm{s}}^{n}, A_{\neg \mathrm{s}}\right) \rightarrow 0$ as $n \rightarrow \infty$.

Next we show that an associative copula can be approximated arbitrarily well w.r.t. $D_{1}$ by a finite ordinal sum consisting only of strict Archimedean copulas or $M$.

Lemma 3.2. Let $C$ be an associative copula. Then for every $\varepsilon>0$ there exist a finite index set $J$, a sequence $\left(A_{j}\right)_{j \in J}$ in $\mathcal{C}_{a r}^{S} \cup\{M\}$ and a partition $\left\{\left[a_{j}, e_{j}\right]\right\}_{j \in J}$ such that

$$
D_{1}\left(C,\left(\left\langle a_{j}, e_{j}, A_{j}\right\rangle\right)_{j \in J}\right) \leq \varepsilon .
$$

Proof. Fix $\varepsilon>0$ and let $C=\left(\left\langle a_{i}, b_{i}, A_{i}\right\rangle\right)_{i \in I}$, where $I \subseteq \mathbb{N}$ is some finite or countably infinite index set. Choose a finite set $J \subseteq I$ such that $\sum_{j \in J} \lambda\left(\left[a_{j}, e_{j}\right]\right) \geq 1-\eta$ for some $\eta>0$. Then $O:=\left(\left\langle a_{j}, e_{j}, A_{j}\right\rangle\right)_{j \in J}$ is a finite ordinal sum of Archimedean copulas with

$$
\begin{aligned}
D_{1}(C, O)= & \int_{[0,1]^{2}}\left|K_{C}(x,[0, y])-K_{O}(x,[0, y])\right| \mathrm{d} \lambda_{2}(x, y) \\
= & \int_{\bigcup_{j \in J}\left[a_{j}, e_{j}\right]^{2}}\left|K_{C}(x,[0, y])-K_{O}(x,[0, y])\right| \mathrm{d} \lambda_{2}(x, y) \\
& +\int_{\quad}\left|K_{C}(x,[0, y])-K_{O}(x,[0, y])\right| \mathrm{d} \lambda_{2}(x, y) \\
& \leq 0+\eta .
\end{aligned}
$$


Choosing $\eta \in(0, \varepsilon)$ we obtain the assertion for arbitrary Archimedean copulas. Moreover, applying Theorem 3.1 to all those Archimedean components that are non-strict completes the proof.

Loosely speaking, we found that selecting only finitely many 'large' components of an associative copula yields a finite associative copula that is close to the original one with respect to $D_{1}$. However, we do not necessarily have a finite ordinal sum purely consisting of Archimedean components as there can still exist subintervals $\left(a_{i}, b_{i}\right)$ of $C$ containing $M$ in our approximation. The following lemma resolves this issue:

Lemma 3.3. For every strict generator $\varphi$ setting $\varphi_{n}:=\varphi^{n}$ and letting $A_{n}$ denote the Archimedean copula with generator $\varphi_{n}$ yields $\lim _{n \rightarrow \infty} D_{1}\left(A_{n}, M\right)=0$.

Proof. We show that for generators of the form $\varphi_{n}(x)=\varphi(x)^{n}$ for some (strict and) continuously differentiable Archimedean generator $\varphi$ we have convergence of the induced sequence $\left(A_{n}\right)_{n \in \mathbb{N}}$ to $M$ w.r.t. $D_{1}$ (see Remark 3.4 for the proof based on a general (strict) generator $\varphi$ ):

Notice that $\varphi_{n}^{-1}(x)=\varphi^{-1}(\sqrt[n]{x}), \varphi_{n}^{\prime}(x)=n \varphi(x)^{n-1} \varphi^{\prime}(x)=n \varphi_{n-1}(x) \varphi^{\prime}(x)$ and the corresponding copula is given by $A_{n}(x, y)=\varphi^{-1}\left(\sqrt[n]{\varphi(x)^{n}+\varphi(y)^{n}}\right)$. It is well-known (see, e.g. [18, Proposition 8.5]) that the sequence $\left(A_{n}\right)_{n \in \mathbb{N}}$ converges uniformly to $M$. In fact, this follows from either of the following two relations:

$$
\begin{aligned}
A_{n}(x, x) & =\varphi^{-1}(\sqrt[n]{2} \cdot \varphi(x)) \stackrel{n \rightarrow \infty}{\longrightarrow} x, \\
F_{A_{n}}^{K}(x) & =x-\frac{1}{n} \frac{\varphi(x)}{\varphi^{\prime}(x)} \stackrel{n \rightarrow \infty}{\longrightarrow} x .
\end{aligned}
$$

The Markov kernel $K_{n}$ corresponding to $A_{n}$ is given by

$$
K_{n}(x,[0, y])=\left\{\begin{array}{ll}
\frac{\varphi^{\prime}(x)}{\varphi^{\prime}\left(A_{n}(x, y)\right)} \cdot \frac{\varphi_{n-1}(x)}{\varphi_{n-1}\left(A_{n}(x, y)\right)} & \text { if } x \in(0,1) \\
1 & \text { if } x \in\{0,1\}
\end{array} .\right.
$$

First suppose $0<x<y<1$. As $\varphi$ is continuously differentiable $\frac{\varphi^{\prime}(x)}{\varphi^{\prime}\left(A_{n}(x, y)\right)}$ tends to 1 as $n \rightarrow \infty$. Concerning the second factor we have

$$
\begin{aligned}
\frac{\varphi_{n-1}(x)}{\varphi_{n-1}\left(A_{n}(x, y)\right)} & =\left(\frac{\varphi(x)}{\varphi\left(A_{n}(x, y)\right)}\right)^{n-1} \cdot \frac{\varphi(x)}{\varphi\left(A_{n}(x, y)\right)} \cdot \frac{\varphi\left(A_{n}(x, y)\right)}{\varphi(x)} \\
& =\frac{\varphi_{n}(x)}{\varphi_{n}\left(A_{n}(x, y)\right)} \cdot \frac{\varphi\left(A_{n}(x, y)\right)}{\varphi(x)} \\
& =\frac{\varphi_{n}(x)}{\varphi_{n}(x)+\varphi_{n}(y)} \cdot \frac{\varphi\left(A_{n}(x, y)\right)}{\varphi(x)} \\
& =\left(1+\left(\frac{\varphi(y)}{\varphi(x)}\right)^{n}\right)^{-1} \cdot \sqrt[n]{1+\left(\frac{\varphi(y)}{\varphi(x)}\right)^{n}} \stackrel{n \rightarrow \infty}{\longrightarrow} 1 \cdot 1=1 .
\end{aligned}
$$

In case of $x>y$ we have $A_{n}(x, y) \rightarrow y$ for $n \rightarrow \infty$ and the first factor of the Markov kernel converges to the constant $\frac{\varphi^{\prime}(x)}{\varphi^{\prime}(y)}$ whereas for the second factor we have $\frac{\varphi(x)}{\varphi\left(A_{n}(x, y)\right)}<1$. Indeed, choose $\delta>0$ such that $0<y<x-\delta$ then there exists $n_{0} \in \mathbb{N}$ such that for all $n \geq n_{0}$ we have $A_{n}(x, y)<x-\delta$ which directly yields that for these $n$

$$
\frac{\varphi(x)}{\varphi\left(A_{n}(x, y)\right)}<\frac{\varphi(x)}{\varphi(x-\delta)}<1
$$

holds. As consequence the second factor converges to 0 as $n \rightarrow \infty$ which shows that for $x \in(0,1)$ we have weak convergence of $K_{n}(x, \cdot) \stackrel{n \rightarrow \infty}{\longrightarrow} K_{M}(x, \cdot)$. Hence $\left(A_{n}\right)_{n \in \mathbb{N}}$ even converges weakly conditional to $M$ which implies converges w.r.t. $D_{1}$ (again see [36]).

\section{Remark 3.4.}

1. Notice that the assumption of $\varphi$ being continuously differentiable is not necessary for Lemma 3.3 to hold. In fact, for some general (strict) Archimedean generator we obtain $\varphi_{n}^{-1}(x)=\varphi^{-1}(\sqrt[n]{x})$ and $D^{+} \varphi_{n}(x)=$ $n \varphi(x)^{n-1} D^{+} \varphi(x)=n \varphi_{n-1}(x) D^{+} \varphi(x)$. We have to show that there exists some $\Lambda \in \mathcal{B}([0,1])$ of full measure 
such that for every $x \in \Lambda$ and $y \in U_{X} \subseteq[0,1]$ with dense set $U_{x}$ we have pointwise convergence of $\left(K_{n}(x,[0, y])\right)_{n \in \mathbb{N}}$ to $K_{M}(x,[0, y])$.

To this end, setting $\Lambda:=\operatorname{Cont}\left(D^{+} \varphi\right)$ yields $\lambda(\Lambda)=1$, and for $x \in \Lambda$ define $U_{x}:=\bigcap_{n \in \mathbb{N}}\{u \in[0,1]$ : $\left.A_{n}(x, u) \in \operatorname{Cont}\left(D^{+} \varphi\right)\right\}$ it follows that $U_{x}$ is the countable intersection of sets of full measure and as such has full measure itself. The rest of the proof is identical to the one of Lemma 3.3 above.

2. Notice that by Lemma 4.3 for $x>y$ we even have weak conditional convergence of general sequences of Archimedean copulas uniformly converging to $M$.

Example 3.5. The (normalized) generator $\varphi(x)=1 / x-1$ produces the copula commonly abbreviated by $A_{\varphi}=\frac{\Pi}{\Sigma-\Pi}$ which is a member of numerous families of Archimedean copulas. Moreover, the generators $\varphi_{n}(x)=$ $\varphi(x)^{n}=(1 / x-1)^{n}$ define the family (4.2.12) in Nelsen [27] and the sequence of induced copulas $\left(A_{n}\right)_{n \in \mathbb{N}}$ not only converges uniformly to $M$, but also weakly conditional by Lemma 3.3 .
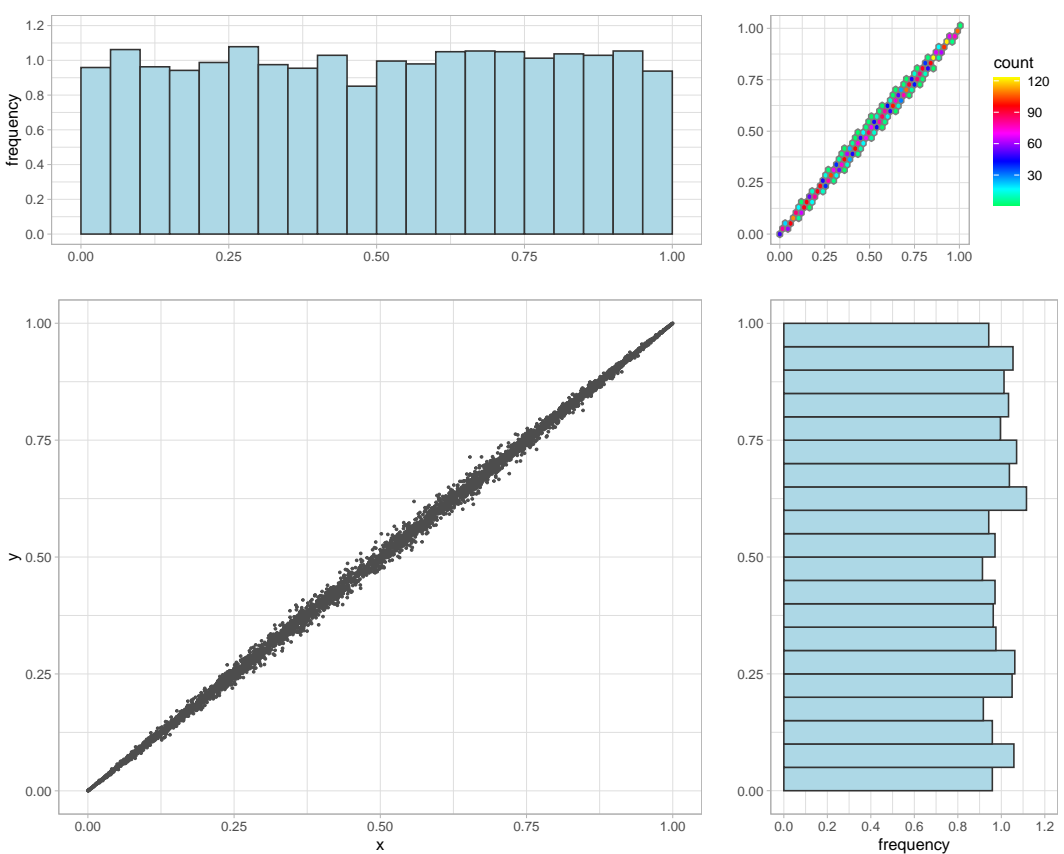

Figure 1: Sample of size 5000 from the copula $A_{n}$ with $n=40$ as considered in Example 3.5 as well as marginal histograms and bivariate histogram.

Theorem 3.6. Let $C$ be an associative copula. Then for every $\varepsilon>0$ there is a finite ordinal sum $A$ of strict Archimedean copulas such that

$$
D_{1}(C, A) \leq \varepsilon
$$

holds.

Proof. Fix $\varepsilon>0$. By Lemma 3.2 there exists a finite ordinal sum $O$ (with strict Archimedean components) such that $D_{1}(C, O)<\frac{\varepsilon}{2}$ holds. Applying Lemma 3.3 we can find some strict $A_{\varepsilon} \in \mathcal{C}_{\text {ar }}$ with $D_{1}\left(A_{\varepsilon}, M\right)<\frac{\varepsilon}{2^{k+1}}$, where $k$ denotes the size of the partition corresponding to $O$. Replacing every segment (along the diagonal) of $O$ containing a shrunk version of $M$ by a shrunk version of $A_{\varepsilon}$ yields another finite ordinal sum $A$ consisting only of (shrunk versions of strict) Archimedean copulas. Altogether we get $D_{1}(C, A) \leq D_{1}(C, O)+D_{1}(O, A) \leq \varepsilon$ which completes the proof. 


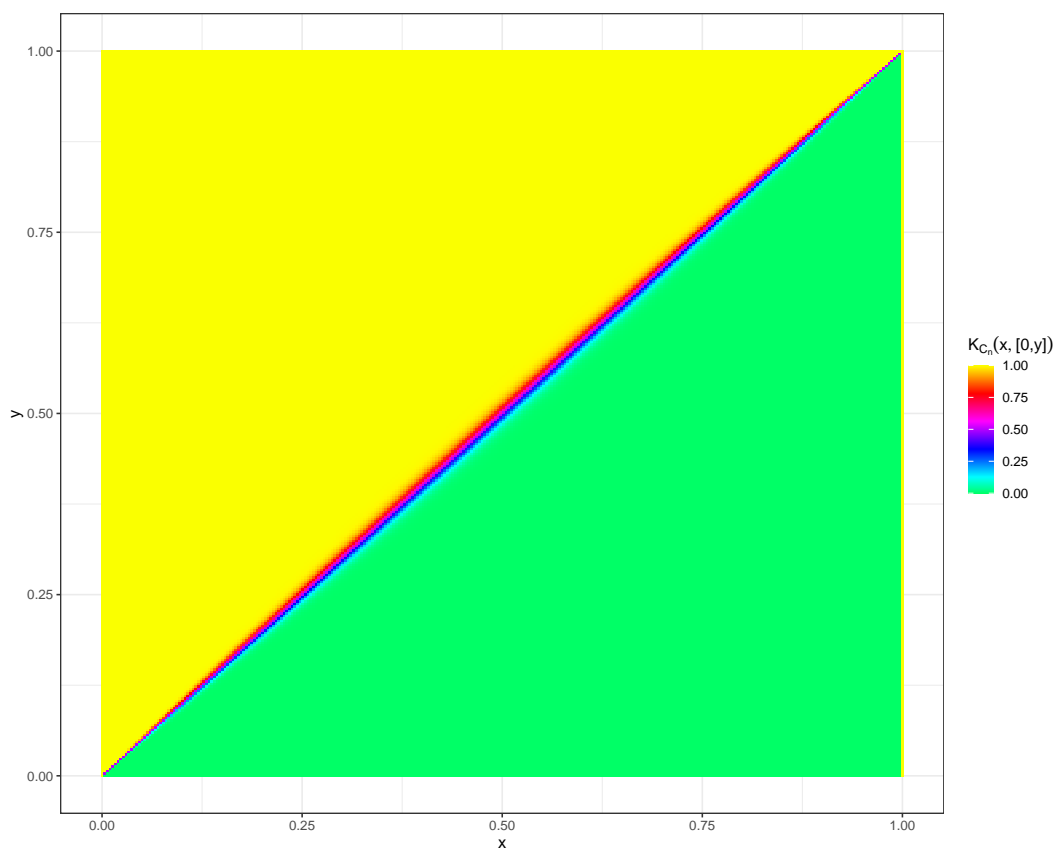

Figure 2: Heatmap of $(x, y) \mapsto K_{A_{n}}(x,[0, y])$ of the copula $A_{n}$ for $n=40$ as considered in Example 3.5.

As next step we show that the ordinal sum $C=\left(\left\langle 0, c, A_{1}\right\rangle,\left\langle c, 1, A_{2}\right\rangle\right)$ of two strict Archimedean copulas can be approximated arbitrarily well with respect to $D_{1}$ by one strict Archimedean copula. In [19] the authors define for every $\varepsilon>0$ a strict Archimedean copula $A_{\varepsilon}$ having the property that $d_{\infty}\left(A_{\varepsilon}, C\right)<\varepsilon$. We prove that for this particular glueing construction even $\lim _{\varepsilon \rightarrow 0} D_{1}\left(A_{\varepsilon}, C\right) \rightarrow 0$ holds. For each $\varepsilon>0$ and $c \in(0,1)$ setting

$$
\varphi_{\varepsilon}(x)= \begin{cases}\varphi_{1}\left(T_{1}(x)\right) & \text { if } x \in\left[0, c-\frac{\varepsilon}{2}\right], \\ g(x) & \text { if } x \in\left(c-\frac{\varepsilon}{2}, x_{0}\right], \\ \frac{g\left(x_{0}\right)}{\varphi_{2}\left(T_{2}\left(x_{0}\right)\right)} \cdot \varphi_{2}\left(T_{2}(x)\right) & \text { if } x \in\left(x_{0}, 1\right]\end{cases}
$$

defines the generator of a strict Archimedean copula $A_{\varepsilon}$ fulfilling $d_{\infty}\left(C, A_{\varepsilon}\right)<\varepsilon$. Here, $\varphi_{1}, \varphi_{2}$ are the generators of $A_{1}, A_{2}$ and $T_{1}, T_{2}$ are the transformations from [0, $\left.c\right]$ and $[c, 1]$ into [0,1], respectively. The linear function $g$ defined on $\left(c-\frac{\varepsilon}{2}, x_{0}\right.$ ] for some $x_{0}<c+\frac{\varepsilon}{2}$ connects the two (appropriately transformed) generators of $A_{1}, A_{2}$ in a way such that the resulting map is again an Archimedean generator. We will make use of this construction, however, the upcoming proof is based on the order of the copulas $C$ and $A_{\varepsilon}$ in different sections of $[0,1]^{2}$ rather than on the formula of $\varphi_{\varepsilon}$.

Example 3.7. Let $\varphi_{1}(x)=-\log (x) / \log (2)$ and $\varphi_{2}(x)=(1 / x-1)^{40}$ then the induced strict Archimedean copulas are $\Pi$ and $A_{40}$ from Example 3.5, respectively. Choosing the partition $\{[0,0.4],[0.4,1]\}$ we obtain an associative copula $C=\left(\left\langle 0,0.4, A_{1}\right\rangle,\left\langle 0.4,1, A_{2}\right\rangle\right)$. Following the construction in [19] and considering $\varepsilon>0$ yields a strict Archimedean copula $A_{\varepsilon}$ fulfilling $d_{\infty}\left(C, A_{\varepsilon}\right)<\varepsilon$.

Lemma 3.8. Let $C=\left(\left\langle 0, c, A_{1}\right\rangle,\left\langle c, 1, A_{2}\right\rangle\right)$ be the ordinal sum of the two strict Archimedean copulas $A_{1}, A_{2}$ with respect to some $c \in(0,1)$ and, for $\varepsilon>0$, let $A_{\varepsilon}$ be the strict Archimedean copula with the afore-mentioned generator $\varphi_{\varepsilon}$. Then

$$
D_{1}\left(C, A_{\varepsilon}\right) \leq 4 \varepsilon
$$

Proof. In order to simplify notation, within integrals we will (sometimes) set $F_{C}^{x}(y)=K_{C}(x,[0, y])$ and $F_{\varepsilon}^{\chi}(y)=K_{\varepsilon}(x,[0, y])$ for the Markov kernels of $C$ and $A_{\varepsilon}$, respectively. 

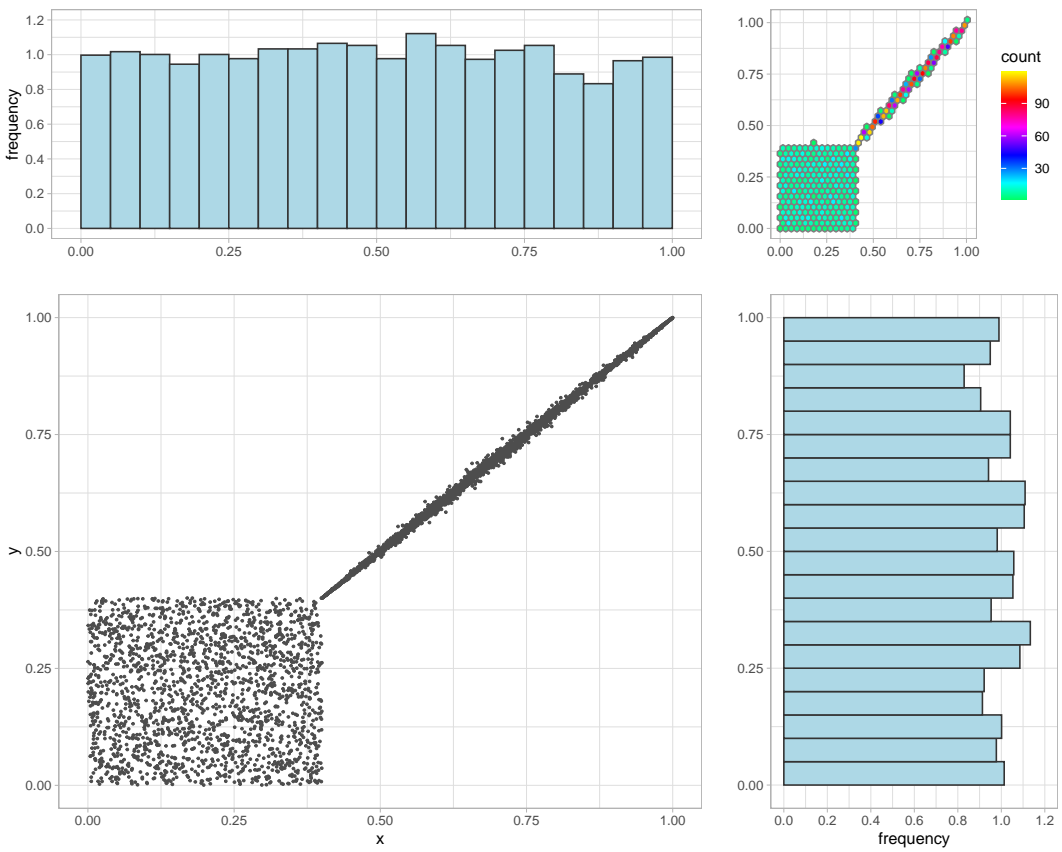

Figure 3: Sample of size 5000 from the Archimedean copula $A_{\varepsilon}$ for $\varepsilon=1 / 1000$ as considered in Example 3.7 as well as marginal histograms and two dimensional histogram.

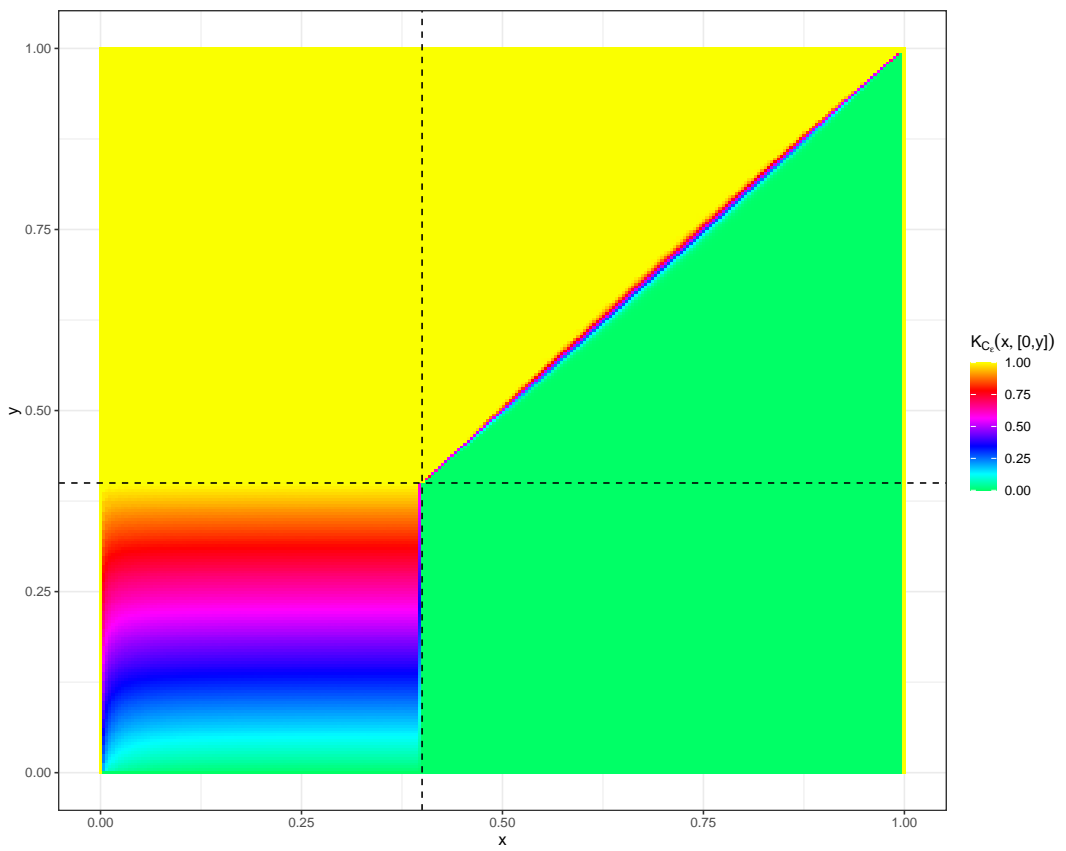

Figure 4: Heatmap of the Markov kernel $K_{A_{\varepsilon}}(x,[0, y])$ of the copula $A_{\varepsilon}$ for $\varepsilon=1 / 1000$ as considered in Example 3.7. 
We start with the following segmentation of $[0,1]^{2}$ and estimate the absolute value within the central horizontal and vertical $\varepsilon$-strips very roughly:

$$
\begin{aligned}
& D_{1}\left(C, A_{\varepsilon}\right)=\int_{[0,1]^{2}}\left|K_{C}(x,[0, y])-K_{\varepsilon}(x,[0, y])\right| \mathrm{d} \lambda_{2}(x, y) \\
& \leq \underbrace{\int_{\left[0, c-\frac{\varepsilon}{2}\right]^{2}}\left|F_{C}^{\chi}(y)-F_{\varepsilon}^{x}(y)\right| \mathrm{d} \lambda_{2}(x, y)}_{\mathrm{I}}+\underbrace{\int_{\left[c+\frac{\varepsilon}{2}, 1\right]^{2}}\left|F_{C}^{x}(y)-F_{\varepsilon}^{x}(y)\right| \mathrm{d} \lambda_{2}(x, y)}_{\mathrm{II}}
\end{aligned}
$$

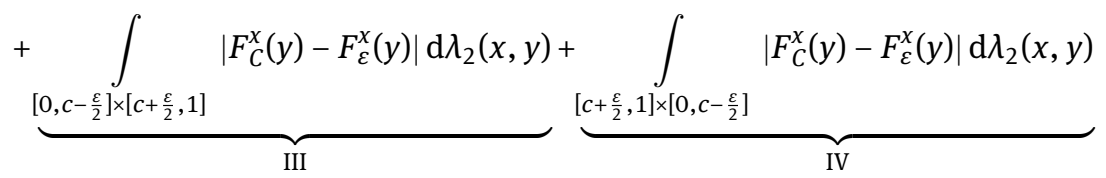

$$
\begin{aligned}
& +\underbrace{\int_{\left(c-\frac{\varepsilon}{2}, c+\frac{\varepsilon}{2}\right) \times[0,1]} 1 \mathrm{~d} \lambda_{2}(x, y)}_{=\varepsilon}+\int_{=\varepsilon}^{\int_{[0,1] \times\left(c-\frac{\varepsilon}{2}, c+\frac{\varepsilon}{2}\right)} 1 \mathrm{~d} \lambda_{2}(x, y)} .
\end{aligned}
$$

The integrals I and II are identical to 0 as in the considered squares $A_{\varepsilon}=C$ and therefore $K_{\varepsilon}=K_{C}$ by Corollary 2.2. Regarding III, for every $(x, y) \in\left[0, c-\frac{\varepsilon}{2}\right] \times\left[c+\frac{\varepsilon}{2}, 1\right]$, we have $1=K_{C}(x,[0, y]) \geq K_{\varepsilon}(x,[0, y])$ and $A_{\varepsilon}(x, y) \in$ $(x-\varepsilon, x]$ so that $x=C(x, y) \geq A_{\varepsilon}(x, y)$. Thus,

$$
\begin{aligned}
\text { III } & =\int_{\left[0, c-\frac{\varepsilon}{2}\right] \times\left[c+\frac{\varepsilon}{2}, 1\right]} K_{C}(x,[0, y])-K_{\varepsilon}(x,[0, y]) \mathrm{d} \lambda_{2}(x, y) \\
& =\int_{\left[c+\frac{\varepsilon}{2}, 1\right]} C\left(c-\frac{\varepsilon}{2}, y\right)-A_{\varepsilon}\left(c-\frac{\varepsilon}{2}, y\right) \mathrm{d} \lambda(y) \leq \varepsilon .
\end{aligned}
$$

Regarding IV, for every $(x, y) \in\left[c+\frac{\varepsilon}{2}, 1\right] \times\left[0, c-\frac{\varepsilon}{2}\right]$, we have $0=K_{C}(x,[0, y]) \leq K_{\varepsilon}(x,[0, y])$ and $A_{\varepsilon}(x, y) \in$ $(y-\varepsilon, y]$ so that $y=C(x, y) \geq A_{\varepsilon}(x, y)$. As direct consequence,

$$
\begin{aligned}
\mathrm{IV} & =\int_{\left[c+\frac{\varepsilon}{2}, 1\right] \times\left[0, c-\frac{\varepsilon}{2}\right]}\left|K_{C}(x,[0, y])-K_{\varepsilon}(x,[0, y])\right| \mathrm{d} \lambda_{2}(x, y) \\
& =\int_{\left(c+\frac{\varepsilon}{2}, 1\right] \times\left[0, c-\frac{\varepsilon}{2}\right]} K_{\varepsilon}(x,[0, y]) \mathrm{d} \lambda_{2}(x, y) \\
& =\int_{\left[0, c-\frac{\varepsilon}{2}\right]} y-A_{\varepsilon}\left(c+\frac{\varepsilon}{2}, y\right) \mathrm{d} \lambda(y) \leq \varepsilon .
\end{aligned}
$$

Summing up, we have shown $D_{1}\left(C, A_{\varepsilon}\right) \leq \varepsilon+\varepsilon+2 \varepsilon=4 \varepsilon$, which completes the proof.

Combining Theorem 3.6 and Lemma 3.8 and using induction on the number of segments shows the following result:

Theorem 3.9. $\mathcal{C}_{a r}$ is dense in $\left(\mathcal{C}_{a}, D_{1}\right)$.

We conclude this section by answering two open problems posed in [6] regarding Baire category results for Archimedean copulas with respect to $D_{1}$. Following [6] or [30, Chapters $1 \& 9$ ] a subset $S$ of a metric space $(\Omega, d)$ is called nowhere dense if the closure of $S$ in $(\Omega, d)$ does not contain any non-empty open ball of $(\Omega, d)$. Moreover, a subset $S$ of $(\Omega, d)$ is said to be meager or of first category if it is a countable union of nowhere dense sets. $S$ is called co-meager if its complement $\Omega \backslash S$ is meager. Additionally, $S$ is of second category if it is not meager. For more background on Baire categories we refer to [30].

In [6] the authors considered the metric spaces $\left(\mathcal{C}, d_{\infty}\right)$ and $\left(\mathcal{C}, D_{1}\right)$ and derived several Baire category results for different subclasses of $\mathcal{C}$. In particular, they showed that $\mathcal{C}_{a r}^{S}$ is co-meager in $\left(\mathcal{C}_{a r}, d_{\infty}\right)$ as well as 
that $\left(\mathcal{C}_{\mathrm{ar}}, d_{\infty}\right)$ is co-meager in $\left(\mathcal{C}_{\mathrm{a}}, d_{\infty}\right)$, and then conjectured that the same results should hold with respect to the stronger metric $D_{1}$. The proof of the first conjecture is a direct consequence of results from [17] whereas denseness of $\mathcal{C}_{\mathrm{ar}}$ in $\left(\mathcal{C}_{\mathrm{a}}, D_{1}\right)$ (as established above) is the key to confirm the second conjecture:

Corollary 3.10. $\mathcal{C}_{a r}^{s}$ is co-meager in $\left(\mathcal{C}_{a r}, D_{1}\right)$.

Proof. Since in $\mathcal{C}_{\text {ar }}$ convergence with respect to $d_{\infty}$ is equivalent to $D_{1}$-convergence both metrics induce the same topology on $\mathcal{C}_{\mathrm{ar}}$. It therefore follows immediately that $\left(\mathcal{C}_{\mathrm{ar}}, D_{1}\right)$ contains the same co-meager subsets as $\left(\mathcal{C}_{\mathrm{ar}}, d_{\infty}\right)$. In particular, $\mathcal{C}_{\mathrm{ar}}^{\mathrm{s}}$ is co-meager in $\left(\mathcal{C}_{\mathrm{ar}}, D_{1}\right)$.

Corollary 3.11. $\mathcal{C}_{a r}$ is co-meager (hence of second category) in $\left(\complement_{a}, D_{1}\right)$.

Proof. As mentioned before, denseness of $\mathcal{C}_{a r}$ in $\left(\mathcal{C}_{\mathrm{a}}, D_{1}\right)$ is the missing piece to derive the desired result. For the sake of completeness we present the adapted proof (cf. [6, Theorem 2.4]) of the statement: We have to show that $\mathcal{C}_{\mathrm{a}} \backslash \mathcal{C}_{\mathrm{ar}}$ is the countable union of nowhere dense subsets. Setting for $k \in \mathbb{N}$

$$
\mathcal{A}_{k}:=\left\{C \in \mathcal{C}_{\mathrm{a}}: \exists x \in\left[\frac{1}{k}, 1-\frac{1}{k}\right] \text { with } C(x, x)=x\right\}
$$

we obviously have $\mathcal{C}_{\mathrm{a}} \backslash \mathcal{C}_{\mathrm{ar}}=\bigcup_{k \in \mathbb{N}} \mathcal{A}_{k}$. Notice that $\mathcal{A}_{k}$ is closed in $\left(C_{\mathrm{a}}, d_{\infty}\right)$ hence also in $\left(\mathcal{C}_{\mathrm{a}}, D_{1}\right)$. Considering that $\mathcal{C}_{a r}$ is dense in $\mathcal{C}_{a}$ w.r.t. $D_{1}, \mathcal{A}_{k}$ cannot contain any non-empty open ball of $\mathcal{C}_{\mathrm{a}}$, i.e. $\mathcal{A}_{k}$ is nowhere dense in $\mathcal{C}_{a}$. As direct consequence $\mathcal{C}_{a} \backslash \mathcal{C}_{a r}$ is the union of nowhere dense subsets and the proof is complete.

In this sense (see [3]), with respect to $D_{1}$ a typical associative copula is Archimedean and a typical Archimedean copula is strict.

\section{Convergence in the class of associative copulas}

In this section we prove the surprising result that within the class of associative copulas, convergence w.r.t. $d_{\infty}$ is equivalent to convergence w.r.t. $D_{1}$.

As already mentioned for $C \in \mathcal{C}_{\mathrm{a}}$ there exist some finite or countably infinite index set $I \subseteq \mathbb{N},\left\{\left(a_{i}, b_{i}\right)\right\}_{i \in I}$ a collection of subintervals forming a 'partition' of $[0,1]$ and a sequence of copulas $\left(A_{i}\right)_{i \in I}$ with $A_{i} \in \mathcal{C}_{\text {ar }}$ or $A_{i}=M$ such that

$$
C(x, y)=T_{i}^{-1}\left(A_{i}\left(T_{i}(x), T_{i}(y)\right)\right)
$$

whenever $(x, y) \in\left(a_{i}, b_{i}\right)^{2}$ and $C(x, y)=M(x, y)$ otherwise; see Section 3. It follows that a Markov kernel of $C$ is given by

$$
K_{C}(x,[0, y])=\left\{\begin{array}{ll}
K_{A_{i}}\left(T_{i}(x),\left[0, T_{i}(y)\right]\right) & \text { if }(x, y) \in\left(a_{i}, b_{i}\right)^{2} \\
K_{M}(x,[0, y]) & \text { otherwise }
\end{array} .\right.
$$

Moreover, for $t \in\left(a_{i}, b_{i}\right)$ with $A_{i} \in \mathcal{C}_{\text {ar }}$ the $t$-level curve of $C$ restricted to $\left(a_{i}, b_{i}\right)^{2}$ is given by

$$
f^{t}(x)=T_{i}^{-1}\left(f_{i}^{T_{i}(t)}\left(T_{i}(x)\right)\right) .
$$

Note that the level curves of associative copulas are convex.

In the sequel we always assume that our points of interest lie in some subinterval $\left(a_{i}, b_{i}\right)$ with $A_{i} \in \mathcal{C}_{\text {ar }}$ and handle the subintervals of $C$ containing $M$ separately later on. Following [10] and defining the set $E_{s, t}^{C}$ for fixed $s, t \in[0,1]$ by

$$
E_{s, t}^{C}:=\left\{(x, y) \in[0,1]^{2}: x \leq s, C(x, y) \leq t\right\}
$$


it follows that for $s, t \in\left(a_{i}, b_{i}\right)$

$$
\mu_{C}\left(E_{s, t}^{C}\right)=a_{i}+\left(b_{i}-a_{i}\right) \cdot \mu_{A_{i}}\left(E_{T_{i}(s), T_{i}(t)}^{A_{i}}\right)
$$

holds and therefore the following formulas follow immediately: For $s, t \in\left(a_{i}, b_{i}\right)$ with $t \leq s$ we have

$$
\mu_{C}\left(E_{s, t}^{C}\right)=t+\left(b_{i}-a_{i}\right) \cdot \frac{\varphi_{i}\left(T_{i}(s)\right)-\varphi_{i}\left(T_{i}(t)\right)}{D^{+} \varphi_{i}\left(T_{i}(t)\right)}
$$

whereas for $s>b_{i}>t>a_{i}$ we have

$$
\mu_{C}\left(E_{s, t}\right)=t-\left(b_{i}-a_{i}\right) \cdot \frac{\varphi_{i}\left(T_{i}(t)\right)}{D^{+} \varphi_{i}\left(T_{i}(t)\right)} .
$$

Denoting by $L_{t}$ the $t$-level set we further have

$$
\mu_{C}\left(L_{t}\right)=\left(b_{i}-a_{i}\right) \cdot\left[\frac{-\varphi_{i}\left(T_{i}(t)\right)}{D^{+} \varphi_{i}\left(T_{i}(t)\right)}+\frac{\varphi_{i}\left(T_{i}(t)\right)}{D^{-} \varphi_{i}\left(T_{i}(t)\right)}\right] .
$$

Moreover, setting $s=b_{i}$ immediately yields a representation for the Kendall distribution function

$$
F_{C}^{K}(t)=T_{i}^{-1}\left(F_{A_{i}}^{K}\left(T_{i}(t)\right)\right)
$$

for $t \in\left(a_{i}, b_{i}\right)$ which will be of use in the sequel.

We now focus on convergence to $M$, or more generally $C \in \mathcal{C}_{\mathrm{cd}}$, and show the following surprising result:

Theorem 4.1. Let $C_{1}, C_{2}, \ldots$ be copulas, $C \in \mathcal{C}_{c d}$ and suppose that $\left(C_{n}\right)_{n \in \mathbb{N}}$ converges uniformly to $C$. Then the following assertions hold:

1. $\left(C_{n}\right)_{n \in \mathbb{N}}$ converges to $C$ w.r.t. $D_{1}$.

2. $\left(C_{n}^{t} * C_{n}\right)_{n \in \mathbb{N}}$ converges to $M$ w.r.t. $d_{\infty}$.

Proof. Considering the $D_{2}$-distance and the definition of the star product (see Section 2) for arbitrary $A, B \in \mathcal{C}$ we have

$$
\begin{aligned}
D_{2}^{2}(A, B) & =\int_{[0,1]^{2}}\left(K_{A}(x,[0, y])-K_{B}(x,[0, y])\right)^{2} \mathrm{~d} \lambda_{2}(x, y) \\
& =\int_{[0,1]} A^{t} * A(y, y)+B^{t} * B(y, y)-2 \cdot A^{t} * B(y, y) \mathrm{d} \lambda(y)
\end{aligned}
$$

Considering that for $C \in \mathcal{e}_{\mathrm{cd}}$ the identity $C^{t} * C=M$ holds and that the star product is continuous in each argument with respect to $d_{\infty}$ (see [8]) it follows that

$$
\begin{aligned}
0 \leq \limsup _{n \rightarrow \infty} D_{2}^{2}\left(C_{n}, C\right) \leq & \limsup _{n \rightarrow \infty} \int_{[0,1]} C_{n}^{t} * C_{n}(s, s) \mathrm{d} \lambda(s)+\int_{[0,1]} M(s, s) \mathrm{d} \lambda(s) \\
& +\limsup _{n \rightarrow \infty}\left(-2 \cdot \int_{[0,1]} C_{n}^{t} * C(s, s) \mathrm{d} \lambda(s)\right) \\
& =\limsup _{n \rightarrow \infty} \int_{[0,1]} \underbrace{C_{n}^{t} * C_{n}(s, s)}_{\leq M(s, s)} \mathrm{d} \lambda(s)-\frac{1}{2} \leq 0
\end{aligned}
$$

implying $\lim _{n \rightarrow \infty} D_{2}^{2}\left(C_{n}, C\right)=0$.

From the first part we already know that $\lim _{n \rightarrow \infty} \int_{[0,1]} C_{n}^{t} * C_{n}(s, s) \mathrm{d} \lambda(s)=\frac{1}{2}$. Letting $\delta_{n}$ denote the diagonal of $C_{n}^{t} * C$ and considering Lipschitz continuity of diagonals it follows that $\delta_{n}$ converges pointwise to the identity function id ${ }_{[0,1]}$. Letting $B_{\delta_{n}}$ denote the Bertino copula with diagonal $\delta_{n}$ (see [11, 12]) yields $B_{\delta_{n}} \leq C_{n}^{t} * C_{n}$ as $B_{\delta_{n}}$ is the smallest symmetric copula with diagonal $\delta_{n}$. Using the fact that $\delta_{n} \rightarrow \operatorname{id}_{[0,1]}$ we have $B_{\delta_{n}} \rightarrow M$ for $n \rightarrow \infty$. Hence considering $C_{n}^{t} * C_{n} \geq B_{\delta_{n}}$ it follows that $C_{n}^{t} * C_{n} \rightarrow M$ uniformly and the proof is complete. 
The second assertion is surprising in so far that the $*$-product is not jointly continuous w.r.t. $d_{\infty}$ (but w.r.t. $D_{1}$ ).

The following Corollary, which is immediate from Theorem 4.1, states the promised equivalence of $d_{\infty}$ and $D_{1}$-convergence whenever the limiting associative copula is the Fréchet-Hoeffding upper bound $M$ :

Corollary 4.2. Let $C_{1}, C_{2}, \ldots$ be associative copulas. Then the following assertions are equivalent:

(a) $\lim _{n \rightarrow \infty} d_{\infty}\left(C_{n}, M\right)=0$.

(b) $\lim _{n \rightarrow \infty} D_{1}\left(C_{n}, M\right)=0$.

Lemma 4.3. Let $A_{1}, A_{2}, \ldots$ be Archimedean copulas. If $\left(A_{n}\right)_{n \in \mathbb{N}}$ converges uniformly to $M$ then for $x>y$ we have

$$
\lim _{n \rightarrow \infty} K_{A_{n}}(x,[0, y])=K_{M}(x,[0, y]) .
$$

Proof. Recall that

$$
\lim _{n \rightarrow \infty} \frac{\varphi_{n}(t)}{D^{+} \varphi_{n}(s)}=0
$$

whenever $s, t \in(0,1)$ with $s \leq t$; see, e.g. [4, Proposition 5].

Now, fix $x>y$ and suppose that $\varepsilon>0$ fulfills $y+\varepsilon<x$. Convexity of $\varphi_{n}$ yields $0 \geq \varphi_{n}(y)-\varphi_{n}(x) \geq D^{+} \varphi_{n}(x) \cdot(y-x)$. Taking the absolute value and dividing by $\left|D^{+} \varphi_{n}(y)\right|$ we obtain

$$
\frac{\varphi_{n}(y)}{\left|D^{+} \varphi_{n}(y)\right|}-\frac{\varphi_{n}(x)}{\left|D^{+} \varphi_{n}(y)\right|} \geq(x-y) \cdot\left|\frac{D^{+} \varphi_{n}(x)}{D^{+} \varphi_{n}(y)}\right| .
$$

As direct consequence of (4.2) we have $\lim _{n \rightarrow \infty} \frac{D^{+} \varphi_{n}(x)}{D^{+} \varphi_{n}(y)}=0$. Considering $\lim _{n \rightarrow \infty} A_{n}(x, y)=y$ there exists some $n_{0} \in \mathbb{N}$ such that for all $n \geq n_{0}$ we have $A_{n}(x, y) \leq y+\varepsilon$, hence

$$
0 \leq \lim _{n \rightarrow \infty} K_{A_{n}}(x,[0, y])=\lim _{n \rightarrow \infty} \frac{D^{+} \varphi_{n}(x)}{D^{+} \varphi_{n}\left(A_{n}(x, y)\right)} \leq \lim _{n \rightarrow \infty} \frac{D^{+} \varphi_{n}(x)}{D^{+} \varphi_{n}(y+\varepsilon)}=0 .
$$

Remark 4.4. As result of the above Lemma, in case of Archimedean copulas converging uniformly to $M$, we have weak conditional convergence whenever $x>y$ in $[0,1]$. It remains an open question whether the result also holds above the diagonal (i.e. for $x<y$ ) which would be key for proving that within $\mathcal{C}_{a}$ we even have equivalence of $d_{\infty}$-convergence, $D_{1}$-convergence and weak conditional convergence.

As next step we consider a sequence $\left(A_{n}\right)_{n \in \mathbb{N}}$ of Archimedean copulas uniformly converging to some associative copula $C$ whereby we focus on studying convergence within Archimedean blocks of $C$. That is, we focus on $(x, y) \in\left(a_{i}, b_{i}\right)^{2}$, for some $i \in I$, with corresponding copula $A_{i} \in \mathcal{C}_{\text {ar }}$ in the 'partition' $\left\{\left[a_{i}, b_{i}\right]\right\}_{i \in I}$ underlying our associative limit $C$. However, since it is not necessary that $A_{i}$ appears in the sequence $\left(A_{n}\right)_{n \in \mathbb{N}}$ we deviate from this notation; subsequently we denote by $\left(a_{\star}, e_{\star}\right)$ the subinterval of interest corresponding to its Archimedean content $A *$ with generator $\varphi *$ in the ordinal sum representation of $C$.

Lemma 4.5. Let $A_{1}, A_{2}, \ldots$ be Archimedean copulas with generators $\varphi_{1}, \varphi_{2}, \ldots$, respectively and let $C \in \mathcal{C}$ be an associative copula such that $\left(A_{n}\right)_{n \in \mathbb{N}}$ converges uniformly to $C$. Then for every subinterval $\left(a_{\star}, b_{\star}\right)$ of $C$ containing an Archimedean copula the following assertions hold:

1. $\lim _{n \rightarrow \infty} F_{A_{n}}^{K}(x)=F_{C}^{K}(x)$ for all $x \in\left(a_{\star}, b_{\star}\right) \cap T_{\star}^{-1}\left(\operatorname{Cont}\left(D^{+} \varphi_{\star}\right)\right)$,

2. $\lim _{n \rightarrow \infty} \frac{\varphi_{n}(x)}{D^{+} \varphi_{n}(x)}=\left(b_{\star}-a_{\star}\right) \cdot \frac{\varphi_{\star}\left(T_{\star}(x)\right)}{D^{+} \varphi_{\star}\left(T_{\star}(x)\right)}$ for all $x \in\left(a_{\star}, b_{\star}\right) \cap T_{\star}^{-1}\left(\operatorname{Cont}\left(D^{+} \varphi_{\star}\right)\right)$,

3. $\lim _{n \rightarrow \infty} \frac{\varphi_{n}(x)}{\varphi_{n}(y)}=\frac{\varphi_{\star}(T \star(x))}{\varphi_{\star}\left(T_{\star}(y)\right)}$ for all $x, y \in\left(a_{\star}, b_{\star}\right)$, 
4. $\lim _{n \rightarrow \infty} \frac{\varphi_{n}(x)}{D^{+} \varphi_{n}(y)}=\left(b_{\star}-a_{\star}\right) \cdot \frac{\varphi_{\star}\left(T_{\star}(x)\right)}{D^{+} \varphi_{\star}\left(T_{\star}(y)\right)}$ for all $x \in\left(a_{\star}, b_{\star}\right), y \in\left(a_{\star}, b_{\star}\right) \cap T_{\star}^{-1}\left(\operatorname{Cont}\left(D^{+} \varphi_{\star}\right)\right)$,

5. $\lim _{n \rightarrow \infty} \frac{D^{+} \varphi_{n}(x)}{D^{+} \varphi_{n}(y)}=\frac{D^{+} \varphi_{\star}\left(T_{\star}(x)\right)}{D^{+} \varphi_{\star}\left(T_{\star}(y)\right)}$ for all $x, y \in\left(a_{\star}, b_{\star}\right) \cap T_{\star}^{-1}\left(\operatorname{Cont}\left(D^{+} \varphi_{\star}\right)\right)$.

Proof. It is well-known that $\lim _{n \rightarrow \infty} d_{\infty}\left(A_{n}, C\right)=0$ implies $F_{A_{n}}^{K}(x) \rightarrow F_{C}^{K}(x)$ pointwise in continuity points of $F_{C}^{K}$. Hence, (1) is a direct consequence of the before established fact that $F_{C}^{K}(x)=T_{\star}^{-1}\left(F_{A_{\star}}^{K}\left(T_{\star}(x)\right)\right)$ and (2) follows immediately. According to [13], an Archimedean generator $\varphi$ fulfills

$$
\varphi(x)=\varphi(y) \cdot \exp \left(\operatorname{sign}(x-y) \int_{\min (x, y)}^{\max (x, y)} \frac{D^{+} \varphi(t)}{\varphi(t)} \mathrm{d} t\right)
$$

for $x \in[0,1]$ and $y \in(0,1)$. Therefore,

$$
\begin{aligned}
\lim _{n \rightarrow \infty} \frac{\varphi_{n}(x)}{\varphi_{n}(y)} & =\lim _{n \rightarrow \infty} \exp \left(\operatorname{sign}(x-y) \int_{\min (x, y)}^{\max (x, y)} \frac{D^{+} \varphi_{n}(t)}{\varphi_{n}(t)} \mathrm{d} t\right) \\
& =\exp \left(\operatorname{sign}(x-y) \int_{\min (x, y)}^{\max (x, y)} \lim _{n \rightarrow \infty} \frac{D^{+} \varphi_{n}(t)}{\varphi_{n}(t)} \mathrm{d} t\right),
\end{aligned}
$$

where the exchange of limit and integral is justified in [4, Proposition 2]. Considering $x>y$, property (2) and using change of coordinates yields

$$
\lim _{n \rightarrow \infty} \frac{\varphi_{n}(x)}{\varphi_{n}(y)}=\exp \left(\int_{[y, x]} \frac{1}{b_{\star}-a_{\star}} \cdot \frac{D^{+} \varphi_{\star}\left(T_{\star}(t)\right)}{\varphi_{\star}\left(T_{\star}(t)\right)} \mathrm{d} t\right)=\frac{\varphi_{\star}\left(T_{\star}(x)\right)}{\varphi_{\star}\left(T_{\star}(y)\right)},
$$

the case $x<y$ follows similarly. This shows (4) and (5) is a consequence of properties (2) and (4).

We now show that for a sequence of Archimedean copulas converging to an associative limit $C$, within the Archimedean blocks of $C$ we have weak convergence of the corresponding Markov kernels on a dense set above the level curve $f^{a_{\star}}$ of $C$. Notice that $f^{a_{*}}(x)=T_{\star}^{-1}\left(f_{\star}^{0}\left(T_{\star}(x)\right)\right), x \in\left(a_{\star}, b_{\star}\right)$ is the $a_{\star}$-level curve of $C$. Hence the following Theorem specifically includes the case of strict Archimedean blocks in $C$ whereas nonstrict blocks are tackled in Theorem 4.9.

Theorem 4.6. Let $A_{1}, A_{2}, \ldots$ be Archimedean copulas with generators $\varphi_{1}, \varphi_{2}, \ldots$ and let $C$ be an associative copula such that $\left(A_{n}\right)_{n \in \mathbb{N}}$ converges uniformly to $C$. Then for every subinterval $\left(a_{\star}, b_{\star}\right)$ of $C$ containing an Archimedean copula $A_{\star} \in \mathcal{C}_{\text {ar }}$ there exists some $\Lambda \in \mathcal{B}\left(\left(a_{\star}, b_{\star}\right)\right)$ of full measure and for $x \in \Lambda$ some set $U_{x} \subseteq\left(f^{a_{*}}(x), b_{\star}\right)$ which is dense in $\left(f^{a_{*}}(x), b_{\star}\right)$ such that for every $x \in \Lambda$ and $y \in U_{x}$ we have

$$
\lim _{n \rightarrow \infty} K_{A_{n}}(x,[0, y])=K_{A_{\star}}\left(T_{\star}(x),\left[0, T_{\star}(y)\right]\right)=K_{C}(x,[0, y]) .
$$

Proof. Suppose that $x \in\left(a_{\star}, b_{\star}\right)$. Then considering $y=T_{\star}^{-1}(1 / 2) \in\left(a_{\star}, b_{\star}\right)$ property (3) of Lemma 4.5 yields

$$
\lim _{n \rightarrow \infty} \frac{\varphi_{n}(x)}{\varphi_{n}\left(T_{\star}^{-1}\left(\frac{1}{2}\right)\right)}=\varphi_{\star}\left(T_{\star}(x)\right) .
$$

Set $\Lambda:=T_{\star}^{-1}\left(\operatorname{Cont}\left(D^{+} \varphi_{\star}\right)\right)$ then $\Lambda$ is of full measure in $\left(a_{\star}, b_{\star}\right)$ and for $x \in \Lambda$ convexity (of $\left.\varphi_{\star}\left(T_{\star}(x)\right)\right)$ implies (see, e.g., [21, 32])

$$
\lim _{n \rightarrow \infty} \frac{1}{\varphi_{n}\left(T_{\star}^{-1}\left(\frac{1}{2}\right)\right)} D^{+} \varphi_{n}(x)=T_{\star}^{\prime}(x) D^{+} \varphi_{\star}\left(T_{\star}(x)\right),
$$

whereby the chain rule is applicable since $T$ is differentiable and increasing. For $x \in \Lambda$ the set $U_{x}:=\{v \in$ $\left.\left(f^{a_{*}}(x), b_{\star}\right): T_{\star}(C(x, v)) \in \operatorname{Cont}\left(D^{+} \varphi_{\star}\right)\right\}$ is of full measure. In fact,

$$
\begin{aligned}
U_{X} & =\left\{v \in\left(f^{a_{*}}(x), b_{\star}\right): A_{\star}\left(T_{\star}(x), T_{\star}(v)\right) \in \operatorname{Cont}\left(D^{+} \varphi_{\star}\right)\right\} \\
& =\left(h_{\star}^{X}\right)^{-1}\left(\operatorname{Cont}\left(D^{+} \varphi_{\star}\right)\right),
\end{aligned}
$$


where $h_{\star}^{\chi}(v):=A_{\star}\left(T_{\star}(x), T_{\star}(v)\right)$ is an increasing homeomorphism from $\left(f^{a^{*}}(x), b_{\star}\right)$ to [0,1] (cf. [27]). Thus, $\left(h_{\star}^{x}\right)^{-1}\left(\operatorname{Cont}\left(D^{+} \varphi_{\star}\right)^{\complement}\right)$ is at most countably infinite whence $\lambda\left(\left(h_{\star}^{x}\right)^{-1}\left(\operatorname{Cont}\left(D^{+} \varphi_{\star}\right)\right)\right)=b_{\star}-f^{a_{*}}(x)$.

Letting $x \in \Lambda, y \in U_{x}$ and invoking continuous convergence of the right-hand derivatives yields

$$
\lim _{n \rightarrow \infty} \frac{1}{\varphi_{n}\left(T_{\star}^{-1}\left(\frac{1}{2}\right)\right)} D^{+} \varphi_{n}\left(A_{n}(x, y)\right)=T_{\star}^{\prime}(C(x, y)) D^{+} \varphi_{\star}\left(T_{\star}(C(x, y))\right)
$$

and we directly obtain

$$
\begin{aligned}
\lim _{n \rightarrow \infty} K_{A_{n}}(x,[0, y]) & =\lim _{n \rightarrow \infty} \frac{D^{+} \varphi_{n}(x)}{D^{+} \varphi_{n}\left(A_{n}(x, y)\right)} \\
& =\lim _{n \rightarrow \infty} \frac{\frac{D^{+} \varphi_{n}(x)}{\varphi_{n}\left(T_{\star}^{-1}\left(\frac{1}{2}\right)\right)}}{\frac{D^{+} \varphi_{n}\left(A_{n}(x, y)\right)}{\varphi_{n}\left(T_{\star}^{-1}\left(\frac{1}{2}\right)\right)}} \\
& =\frac{T_{\star}^{\prime}(x) D^{+} \varphi_{\star}\left(T_{\star}(x)\right)}{T_{\star}^{\prime}(C(x, y)) D^{+} \varphi_{\star}(T \star(C(x, y)))} \\
& =\frac{D^{+} \varphi_{\star}(T \star(x))}{D^{+} \varphi_{\star}\left(A_{\star}\left(T_{\star}(x), T_{\star}(y)\right)\right)}=K_{C}(x,[0, y])
\end{aligned}
$$

since obviously $T_{\star}^{\prime}(x)=T_{\star}^{\prime}(C(x, y))=\frac{1}{b_{\star}-a_{\star}}$.

According to results of [36] convergence with respect to $D_{1}$ (within $\left(a_{\star}, e_{\star}\right)$ ) follows immediately.

Since every Archimedean copula is an ordinal sum with only trivial idempotent elements, the convergence results from Lemma 4.5 and Theorem 4.6 can be seen as generalizations of [17, Theorem 4.2]:

Theorem 4.7. Let $A, A_{1}, A_{2}, \ldots$ be Archimedean copulas with generators $\varphi, \varphi_{1}, \varphi_{2}, \ldots$ such that $\left(A_{n}\right)_{n \in \mathbb{N}}$ converges uniformly to $A$. Then there exists some $\Lambda \in \mathcal{B}((0,1))$ of full measure and some set $U_{x} \subseteq\left(f^{0}(x), 1\right)$ which is dense in $\left(f^{0}(x), 1\right)$ such that for every $x \in \Lambda$ and $y \in U_{x}$ we have

$$
\lim _{n \rightarrow \infty} K_{A_{n}}(x,[0, y])=K_{A}(x,[0, y]) .
$$

Remark 4.8. At this point we have a very strong convergence property for the following special case of convergence within $\mathcal{C}_{\mathrm{a}}$ : Let $A_{1}, A_{2}, \ldots$ be a sequence of Archimedean copulas converging uniformly to some finite associative copula $C$ having no sections containing $M$, i.e. a finite ordinal sum purely consisting of Archimedean copulas. Then $\left(A_{n}\right)_{n \in \mathbb{N}}$ converges weakly conditional to $C$. This includes the frequently presented case of Archimedean copulas converging to ordinal sums of $W$ (see, e.g., [4],[19]), also called "prototypes" in [34].

Using the afore-mentioned results we can now finally prove the equivalence of convergence with respect to $d_{\infty}$ and convergence with respect to $D_{1}$ within the class of associative copulas:

Theorem 4.9. Let $C, C_{1}, C_{2}, \ldots$ be associative copulas. If the sequence $\left(C_{n}\right)_{n \in \mathbb{N}}$ converges uniformly to $C$ then the sequence even converges with respect to $D_{1}$.

Proof. As proved in Section 3 the class $\mathcal{C}_{\mathrm{ar}}^{\mathrm{s}}$ of strict Archimedean copulas is dense in $\left(\mathcal{C}_{\mathrm{a}}, D_{1}\right)$. Consequently, for every $C_{n}$ there exists some $A_{n} \in \mathcal{C}_{\text {ar }}^{\mathrm{s}}$ such that $D_{1}\left(C_{n}, A_{n}\right)<\frac{1}{n}$. Thus,

$$
D_{1}\left(C_{n}, C\right) \leq D_{1}\left(C_{n}, A_{n}\right)+D_{1}\left(A_{n}, C\right)<\frac{1}{n}+D_{1}\left(A_{n}, C\right)
$$

and it suffices to show that $D_{1}\left(A_{n}, C\right) \rightarrow 0$ for $n \rightarrow \infty$ which can be done as follows: According to [6, Lemma 3] $d_{\infty}\left(A_{n}, C\right) \leq 2 \cdot \sqrt{D_{1}\left(A_{n}, C_{n}\right)}+d_{\infty}\left(C_{n}, C\right)$ and the latter converges to 0 for $n \rightarrow \infty$ whence $\left(A_{n}\right)_{n \in \mathbb{N}}$ converges uniformly to $C$. Write $C=\left(\left\langle a_{i}, b_{i}, B_{i}\right\rangle\right)_{i \in I}$ for some finite or countably infinite index set $I$. Fix $\varepsilon>0$ then as in Lemma 3.2 we can choose a finite set $J \subseteq I$ such that $\sum_{j \in J} \lambda\left(\left(a_{j}, b_{j}\right)\right) \geq 1-\varepsilon$ and consider the following partition of $[0,1]^{2}$ (as before we set $F_{n}^{x}(y)=K_{n}(x,[0, y])=K_{A_{n}}(x,[0, y])$ and $\mu_{n}=\mu_{A_{n}}$, similarly for the limit C): 


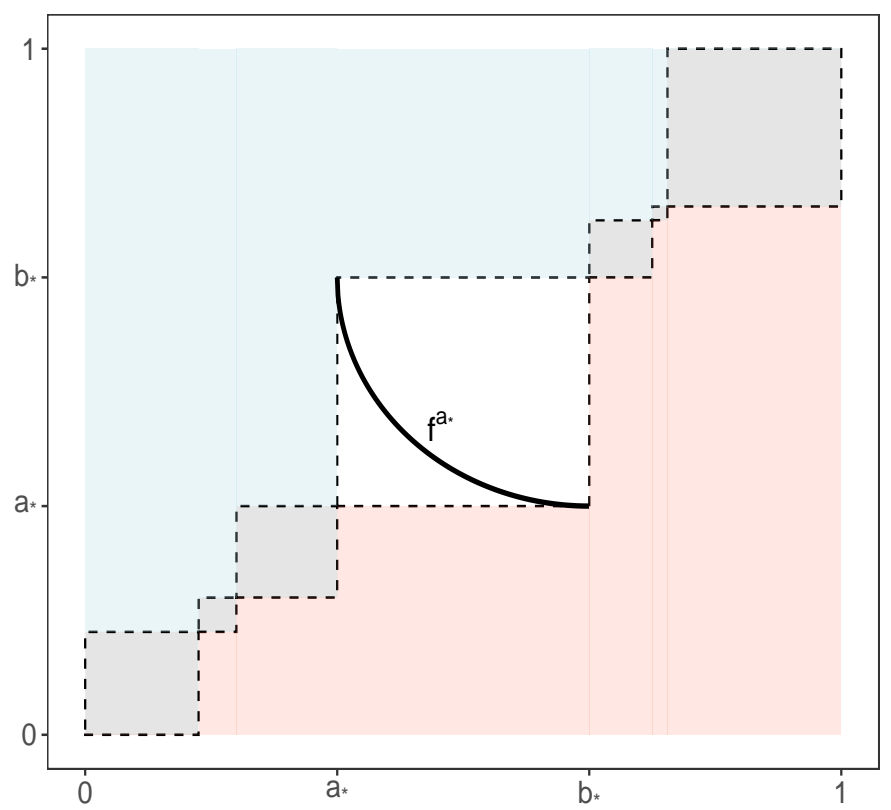

Figure 5: Illustration of the (support of the) associative limit $C$ with $a_{\star}$-level curve $f^{a_{\star}}(x)$ for $x \in\left(a_{\star}, b_{\star}\right)$. The regions $U$ and $L$ considered in the proof of Theorem 4.9 are colorized in light blue and light red, respectively.

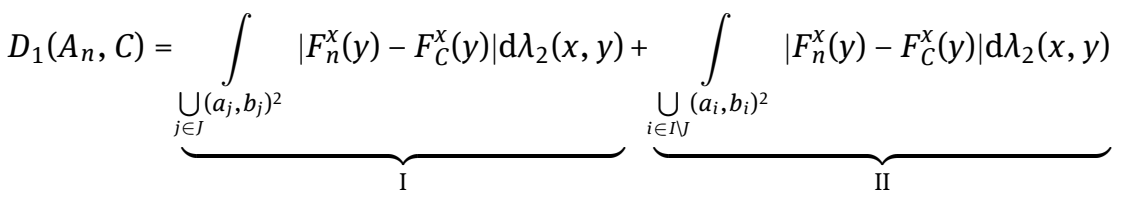

$$
\begin{aligned}
& +\underbrace{\int_{[0,1]^{2} \backslash \bigcup_{i \in I}\left(a_{i}, b_{i}\right)^{2}}\left|F_{n}^{x}(y)-F_{C}^{x}(y)\right| \mathrm{d} \lambda_{2}(x, y)}_{\text {III }}
\end{aligned}
$$

Clearly II $\leq \varepsilon$; for calculating III divide the integration area in the sets $L$ and $U$ denoting the lower and upper part, respectively, i.e. $U=\bigcup_{i \in I}\left(a_{i}, b_{i}\right) \times\left(b_{i}, 1\right)$ and $L=\bigcup_{i \in I}\left(a_{i}, b_{i}\right) \times\left(0, a_{i}\right)$ (as depicted in Figure 5). Using disintegration we get

$$
\begin{aligned}
\int_{U} \mid K_{n}(x,[0, y]) & -K_{C}(x,[0, y]) \mid \mathrm{d} \lambda_{2}(x, y)=\int_{U} 1-K_{n}(x,[0, y]) \mathrm{d} \lambda_{2}(x, y) \\
& =\int_{U} K_{n}(x,(y, 1]) \mathrm{d} \lambda_{2}(x, y) \\
& =\sum_{i \in I} \int_{\left(b_{i}, 1\right)} \underbrace{\mu_{n}\left(\left(a_{i}, b_{i}\right) \times(y, 1]\right)}_{\leq \mu_{n}\left(\left(a_{i}, b_{i}\right) \times\left(b_{i}, 1\right)\right)} \mathrm{d} \lambda(y) \\
& \leq \sum_{i \in I} \mu_{n}\left(\left(a_{i}, b_{i}\right) \times\left(b_{i}, 1\right)\right) \cdot\left(1-b_{i}\right) \\
& \leq \sum_{i \in I} \mu_{n}\left(\left(a_{i}, b_{i}\right) \times\left(b_{i}, 1\right)\right)=\mu_{n}(U)
\end{aligned}
$$

and as $U$ is a $\mu_{C}$-continuity set it follows that $\lim _{n \rightarrow \infty} \mu_{n}(U)=\mu_{C}(U)=0$. The lower area $L$ follows analogously whence there is $n_{0} \in \mathbb{N}$ such that III $\leq \varepsilon$ for all $n \geq n_{0}$.

It remains to show that the same holds true for I: We split the index set $J$ into $J_{1}$ denoting the subset of indices 
corresponding to blocks containing an Archimedean copula and $J_{2}$ corresponding to blocks containing $M$, i.e.

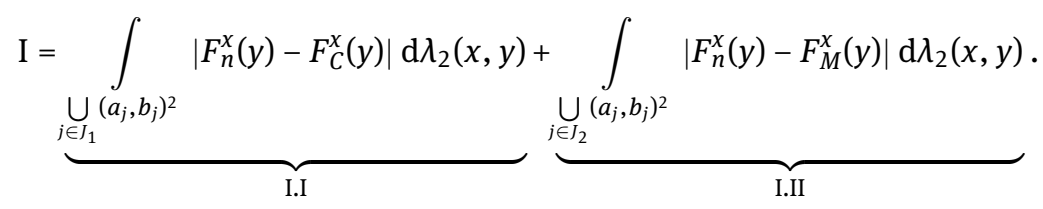

We begin with the Archimedean case I.I: To facilitate reading we focus on a single subinterval $\left(a_{\star}, b_{\star}\right):=$ $\left(a_{j}, b_{j}\right)$ for some $j \in J_{1}$ containing an Archimedean copula. Then

$$
\begin{aligned}
& \int_{\left(a_{*}, b_{*}\right)^{2}}\left|F_{n}^{x}(y)-F_{C}^{x}(y)\right| \mathrm{d} \lambda(x) \mathrm{d} \lambda(y) \\
& =\int_{\left(a_{*}, b_{*}\right)} \int_{\left\{x<f^{a_{*}}(y)\right\}}\left|F_{n}^{x}(y)-F_{C}^{x}(y)\right| \mathrm{d} \lambda(x) \mathrm{d} \lambda(y)+\int_{\left(a_{*}, b_{*}\right)} \int_{\left\{x \geq f^{a_{*}}(y)\right\}}\left|F_{n}^{x}(y)-F_{C}^{x}(y)\right| \mathrm{d} \lambda_{2}(x, y),
\end{aligned}
$$

where for fixed $y \in\left(a_{\star}, b_{\star}\right)$ the set $\left\{x<f^{a_{\star}}(y)\right\}=\left\{x \in\left(a_{\star}, b_{\star}\right): x<f^{a_{\star}}(y)\right\}=\left(a_{\star}, f^{a_{*}}(y)\right)$. The first integrand fulfills $F_{C}^{x}(y)=K_{C}(x,[0, y])=0$ and we get

$$
\int_{\left(a_{*}, b_{*}\right)} \int_{\left\{x<f^{a_{*}}(y)\right\}} K_{n}(x,[0, y]) \mathrm{d} \lambda_{2}(x, y)=\int_{\left(a_{*}, b_{*}\right)} \mu_{n}\left(\left(a_{\star}, f^{a_{*}}(y)\right) \times[0, y]\right) \mathrm{d} \lambda(y)
$$

which converges to $\int_{\left(a_{\star}, b_{\star}\right)} \mu_{C}\left(\left(a_{\star}, f^{a_{*}}(y)\right) \times[0, y]\right) \mathrm{d} \lambda(y)=0$ for $n \rightarrow \infty$ by Dominated Convergence. For the second summand we have

$$
\int_{\left(a_{*}, b_{*}\right)} \int_{\left\{x \geq f^{a_{*}}(y)\right\}}\left|F_{n}^{x}(y)-F_{C}^{x}(y)\right| \mathrm{d} \lambda_{2}(x, y)=\int_{\left(a_{*}, b_{*}\right)} \int_{\left\{y \geq f^{a_{*}}(x)\right\}}\left|F_{n}^{x}(y)-F_{C}^{x}(y)\right| \mathrm{d} \lambda_{2}(y, x) .
$$

Observe that now we are in the setting of Theorem 4.6 and hence also have convergence to 0 for $n \rightarrow \infty$. To calculate I.II we proceed similarly, fix a subinterval $\left(\bar{a}_{\star}, \bar{b}_{\star}\right):=\left(a_{j}, b_{j}\right)$ for some $j \in J_{2}$ of $C$ containing a copy of $M$ and consider

$$
\begin{aligned}
\int_{\left(\bar{a}_{*}, \bar{b}_{*}\right)^{2}} \mid K_{n}(x,[0, y]) & -K_{M}(x,[0, y]) \mid \mathrm{d} \lambda_{2}(x, y) \\
& =\int_{\left(\bar{a}_{*}, \bar{b}_{*}\right)}\left\{\int_{\left(\bar{a}_{*}, y\right]} 1-K_{n}(x,[0, y]) \mathrm{d} \lambda(x)+\int_{\left[y, \bar{b}_{*}\right)} K_{n}(x,[0, y]) \mathrm{d} \lambda(x)\right\} \mathrm{d} \lambda(y) \\
& =\int_{\left(\bar{a}_{*}, \bar{b}_{*}\right)} \mu_{n}\left(\left(\bar{a}_{*}, y\right] \times(y, 1]\right)+\mu_{n}\left(\left[y, \bar{b}_{\star}\right) \times[0, y]\right) \mathrm{d} \lambda(y) \\
& \rightarrow \int_{\left(\bar{a}_{*}, \bar{b}_{*}\right)} \mu_{C}\left(\left(\bar{a}_{*}, y\right] \times(y, 1]\right)+\mu_{C}\left(\left[y, \bar{b}_{\star}\right) \times[0, y]\right) \mathrm{d} \lambda(y)=0
\end{aligned}
$$

for $n \rightarrow \infty$ (again using Dominated Convergence). It directly follows that there exists $n_{1} \in \mathbb{N}$ such that for all $n \geq n_{1}$ we have $\mathrm{I} \leq \varepsilon$.

Altogether, there exists $n_{2} \in \mathbb{N}$ such that for all $n \geq n_{2}=\max \left(n_{0}, n_{1}\right)$ we have

$$
D_{1}\left(A_{n}, C\right)=\mathrm{I}+\mathrm{II}+\mathrm{III} \leq 3 \cdot \varepsilon,
$$

which completes the proof. 


\section{Acknowledgements}

The first author gratefully acknowledges the financial support from Porsche Holding Austria and Land Salzburg within the WISS 2025 project KFZ (P1900123). Moreover, the second and the third author gratefully acknowledge the support of the WISS 2025 project 'IDA-lab Salzburg' (20204-WISS/225/197-2019 and 0102F1901166-KZP).

Conflict of interest statement: Authors state no conflict of interest.

\section{References}

[1] Alsina, C., B. Schweizer, and M. Frank (2006). Associative Functions. World Scientific Publishing, Singapore.

[2] Billingsley, P. (1999). Convergence of Probability Measures. Second edition. John Wiley \& Sons, New York.

[3] Bruckner, A. M, J. B. Bruckner, and B. S. Thomson (2008). Real Analysis. Second edition. Available at http:// classicalrealanalysis.info/com/Real-Analysis.php.

[4] Charpentier, A. and J. Segers (2008). Convergence of Archimedean copulas. Statist. Probab. Lett. 78(4), 412-419.

[5] Darsow, W. F., B. Nguyen, and E. T. Olsen (1992). Copulas and Markov processes. Illinois J. Math. 36(4), 600-642.

[6] Durante, F., J. Fernández-Sánchez, and W. Trutschnig (2016). Baire category results for exchangeable copulas. Fuzzy Sets Syst. 284, 146-151.

[7] Durante, F. and P. Jaworski (2010). A New Characterization of Bivariate Copulas. Comm. Statist. Theory Methods 39, 29012912.

[8] Durante, F. and C. Sempi (2016). Principles of Copula Theory. CRC Press, Boca Raton FL.

[9] Fernández-Sánchez, J. and W. Trutschnig (2015a). Conditioning based metrics on the space of multivariate copulas and their interrelation with uniform and levelwise convergence and Iterated Function Systems. J. Theoret. Probab. 28, 1311-1336.

[10] Fernández-Sánchez, J. and W. Trutschnig (2015b). Singularity aspects of Archimedean copulas. J. Math. Anal. Appl. 432(1), 103-113.

[11] Fernández-Sánchez, J. and W. Trutschnig (2016). Some members of the class of (quasi-)copulas with given diagonal from the Markov kernel perspective. Comm. Statist. Theory Methods 45(5), 1508-1526.

[12] Fredricks, G. A. and R. B. Nelsen (2002). The Bertino family of copulas. In Cuadras C.M., Fortiana J., Rodriguez-Lallena J.A. (Eds.), Distributions With Given Marginals and Statistical Modelling, pp. 81-91. Springer, Dordrecht.

[13] Genest, C. and L.-P. Rivest (1993). Statistical inference procedures for bivariate Archimedean copulas. J. Amer. Statist. Assoc. 88(423), 1034-1043.

[14] Griessenberger, F., J. Fernández-Sánchez, and W. Trutschnig (2021). Some properties of double shuffles of bivariate copulas and (extreme) copulas invariant with respect to Lüroth double shuffles. Fuzzy Sets Syst., to appear. Available at https: //doi.org/10.1016/j.fss.2021.02.014.

[15] Kallenberg, O. (2002). Foundations of Modern Probability. Springer, New York.

[16] Kannan, R. and C. K. Krueger (1996). Advanced Analysis. Springer, New York.

[17] Kasper, T., S. Fuchs, and W. Trutschnig (2020). On weak conditional convergence of bivariate Archimedean and extreme value copulas, and consequences to nonparametric estimation. Bernoulli, to appear. Available at http://www.bernoullisociety.org/index.php/publications/bernoulli-journal/bernoulli-journal-papers.

[18] Klement, E. P., R. Mesiar, and E. Pap (2000). Triangular Norms. Springer, Dordrecht.

[19] Klement, E. P., R. Mesiar, and E. Pap (2001). Uniform approximation of associative copulas by strict and non-strict copulas. Illinois J. Math. 45(4), 1393-1400.

[20] Klenke, A. (2008). Wahrscheinlichkeitstheorie. Springer, Berlin.

[21] Kosmol, P. (2010). Optimierung und Approximation. De Gruyter, Berlin.

[22] Lancaster, H. O. (1963). Correlation and complete dependence of random variables. Ann. Math. Statist. 34(4), $1315-1321$.

[23] Ling, C.-H. (1965). Representation of associative functions. Publ. Math. Debrecen 12, 189-212.

[24] Li, X., P. Mikusinski, M.D. Taylor (1998). Strong approximation of copulas. J. Math. Anal. Appl. 255(2), 608-623

[25] Moynihan, R. (1978). On $\tau_{T}$ semigroups of probability distribution functions II. Aequationes Math. 17, 19-40.

[26] Mroz, T., S. Fuchs, W. Trutschnig (2021). How simplifying and flexible is the simplifying assumption in pair-copula constructions - analytic answers in dimension three and a glimpse beyond. Electron. J. Stat. 15, 1951-1992

[27] Nelsen, R. B. (2006). An Introduction to Copulas. Second edition. Springer, New York.

[28] Nelsen, R. B., J. J. Quesada-Molina, J. A. Rodríguez-Lallena, and M. Úbeda Flores (2009). Kendall distribution functions and associative copulas. Fuzzy Sets Syst. 160(1), 52-57.

[29] Olsen, E.T., W.F. Darsow, B. Nguyen (1996). Copulas and Markov operators, in L. Rüschendorf, B. Schweizer, and M.D. Taylor (Eds.), Distributions with Fixed Marginals and Related Topics, pp. 244-259. Institute od Mathematical Statistics, Hayward CA. 
[30] Oxtoby, J. C. (1980). Measure and Category. Springer, New York.

[31] Pollard, D. (2001). A User's Guide to Measure Theoretic Probability. Cambridge University Press.

[32] Rockafeller, R.T. (1972). Convex Analysis. Princeton University Press.

[33] Rudin, W. (1976). Principles of Mathematical Analysis. McGraw-Hill, Auckland.

[34] Schreyer, M., R. Paulin, and W. Trutschnig (2017). On the exact region determined by Kendall's $\tau$ and Spearman's $\rho$. J. $R$. Stat. Soc. Ser. B Stat. Methodol. 79(2), 613-633.

[35] Spanhel, F. and M. Kurz (2019). Simplified vine copula models: Approximations based on the simplifying assumption. Electron. J. Stat. 13, 1254-1291.

[36] Trutschnig, W. (2011). On a strong metric on the space of copulas and its induced dependence measure. J. Math. Anal. Appl. 384(2), 690-705.

[37] Trutschnig, W. (2012). Some results on the convergence of (quasi-) copulas. Fuzzy Sets Syst. 191, 113-121.

[38] Trutschnig, W. and J. Fernández-Sánchez (2012). Idempotent and multivariate copulas with fractal support. J. Statist. Plann. Inference 142(12), 3086-3096. 